\title{
Therapeutic Efficiency of Multiple Applications of Magnetic Hyperthermia Technique in Glioblastoma Using Aminosilane Coated Iron Oxide Nanoparticles: In Vitro and In Vivo Study
}

\author{
Gabriel N. A. Rego ${ }^{1}{ }^{1}$, Mariana P. Nucci ${ }^{2}{ }^{\circledR}$, Javier B. Mamani ${ }^{1}$, Fernando A. Oliveira ${ }^{1}$, \\ Luciana C. Marti ${ }^{1}$ (D) Igor S. Filgueiras ${ }^{1}$, João M. Ferreira ${ }^{1}$, Caroline C. Real ${ }^{3}$, \\ Daniele de Paula Faria $^{3}$, Paloma L. Espinha ${ }^{1}$, Daianne M. C. Fantacini ${ }^{4}$ D, Lucas E. B. Souza $^{4}$, \\ Dimas T. Covas ${ }^{4}$, Carlos A. Buchpiguel ${ }^{3}$ and Lionel F. Gamarra ${ }^{1, * \mathbb{D}}$ \\ 1 Hospital Israelita Albert Einstein, Sao Paulo 05652-900, Brazil; gabriel.nery@einstein.br (G.N.A.R.); \\ javierbm@einstein.br (J.B.M.); fernando.anselmo@einstein.br (F.A.O.); luciana.marti@einstein.br (L.C.M.); \\ igor.filgueiras@usp.br (I.S.F.); joãomatiasferreirav@gmail.com (J.M.F.); palomaespinha10@gmail.com (P.L.E.) \\ 2 Laboratory of Magnetic resonance (LIM-44), Faculdade de Medicina, Universidade de Sao Paulo, \\ Sao Paulo 01246-903, SP, Brazil; mariana.nucci@hc.fm.usp.br \\ 3 Laboratory of Nuclear Medicine (LIM-43), Departamento de Radiologia e Oncologia, Faculdade de \\ Medicina, Universidade de Sao Paulo, Sao Paulo 01246-903, SP, Brazil; caroline.real@hc.fm.usp.br (C.C.R.); \\ daniele.faria@hc.fm.usp.br (D.d.P.F.); buch@usp.br (C.A.B.) \\ 4 Faculdade de Medicina de Ribeirao Preto, Universidade de Sao Paulo, Ribeirao Preto, Sao Paulo 4049-900, \\ Brazil; daianne.carvalho@hemocentro.fmrp.usp.br (D.M.C.F.); lucasebsouza@usp.br (L.E.B.S.); \\ dimas@fmrp.usp.br (D.T.C.) \\ * Correspondence: lionelgamarra7@gmail.com; Tel.: +55-11-2151-0243
}

Received: 21 December 2019; Accepted: 17 January 2020; Published: 31 January 2020 updates

\begin{abstract}
Magnetic hyperthermia (MHT) has been shown as a promising alternative therapy for glioblastoma (GBM) treatment. This study consists of three parts: The first part evaluates the heating potential of aminosilane-coated superparamagnetic iron oxide nanoparticles (SPIONa). The second and third parts comprise the evaluation of MHT multiple applications in GBM model, either in vitro or in vivo. The obtained heating curves of SPIONa $(100 \mathrm{~nm},+20 \mathrm{mV})$ and their specific absorption rates (SAR) stablished the best therapeutic conditions for frequencies ( $309 \mathrm{kHz}$ and $557 \mathrm{kHz}$ ) and magnetic field (300 Gauss), which were stablished based on three in vitro MHT application in C6 GBM cell line. The bioluminescence (BLI) signal decayed in all applications and parameters tested and $309 \mathrm{kHz}$ with 300 Gauss have shown to provide the best therapeutic effect. These parameters were also established for three MHT applications in vivo, in which the decay of BLI signal correlates with reduced tumor and also with decreased tumor glucose uptake assessed by positron emission tomography (PET) images. The behavior assessment showed a slight improvement after each MHT therapy, but after three applications the motor function displayed a relevant and progressive improvement until the latest evaluation. Thus, MHT multiple applications allowed an almost total regression of the GBM tumor in vivo. However, futher evaluations after the therapy acute phase are necessary to follow the evolution or tumor total regression. BLI, positron emission tomography (PET), and spontaneous locomotion evaluation techniques were effective in longitudinally monitoring the therapeutic effects of the MHT technique.
\end{abstract}

Keywords: magnetic hyperthermia; glioblastoma; SPION; nanoparticle; aminosilane; bioluminescence; PET/CT; motor behavior 


\section{Introduction}

Glioblastomas (GBM) comprise a group of primary or secondary brain malignant tumors [1,2], which are the most aggressive and frequent tumor (55.4\% of gliomas) of the central nervous system, and were classified by the WHO as level IV [3]. The GBM conventional treatment consists of tumor surgical resection followed by concomitant administration of radiotherapy and chemotherapy with temozolomide (TMZ). The treatment in most cases is palliative, non-curative, and only provides a short increase in survival for patients, which usually does not exceed 24 months [4,5]. These limitations justify decades of commitment in preclinical researches, pursuing alternatives and more effective therapies for GBM treatment.

Among these therapies, there is the magneto hyperthermia therapy (MHT) that has been improved and studied in vitro and in vivo, alone or combined with the traditional therapeutic protocols. MHT is a procedure that releases heat locally in the tumor by mediators such as magnetic nanoparticles (MNP) by exposure to an alternating magnetic field (AMF) with appropriate frequency and magnetic field strength [6]. The most commonly used MNP in this technique is the superparamagnetic iron oxide nanoparticle (SPION). SPION belongs to materials classified as ferrimagnetic because they have a core with a crystal structure of hematite $\left(\mathrm{Fe}_{2} \mathrm{O}_{3}\right)$, magnetite $\left(\mathrm{Fe}_{3} \mathrm{O}_{4}\right)$, maghemite $\left(\gamma-\mathrm{Fe}_{2} \mathrm{O}_{3}\right)$ or mixed ferrites $[7,8]$. In addition, this material possesses a magnetization called superparamagnetism, which depends on their physicochemical characteristics, such as diameter, morphology, crystal structure, among others. SPION are characterized by the formation of highly magnetic moments when exposed to AMF, which completely disappears when AMF is turned off $[9,10]$.

Another important point is the SPION coating with organic or inorganic molecules such as dextran, chitosan, silica, starch, and polydimethylamine, which can alter SPION biodistribution, toxicity, clearance, surface charge, colloidal stability, cellular uptake, among other functions [11]. A SPION possible coating is the aminosilane, a material known to enhance protein and cell adhesion [9]. Aminosilane also display low toxicity and has been widely used in the construction of complex and multifunctional drug delivery systems [12]. Some important characteristics of SPION coated with aminosilane (SPIONa) for the MHT application is their higher uptake by cells [9], their previous implication in preclinical studies [13-17], and referenced use in several phase I and II clinical trials [18-21].

The greatest challenges for therapeutic techniques to achieve efficiency are based on parameters optimization, equipment configuration and delivery system including NPM type, physicochemical characteristics, dose, and number of applications that is needed for tumoral tissue eradication. Similarly occurs with the MHT technique, where studies describe comparative analysis in order to establish the best parameters of AMF, periodicity and MHT fractionation [14,15,22-26]. Preclinical studies report a variety of parameters depending on tumor type, tumor localization, magnetic nanomaterial type and miscellaneous AMF configurations $[13-15,22,23,25,26]$. These variations have lead to difficulties in finding comparative parameters for results normalization, which requires analysis of specific absorption rate (SAR) and calibration protocols to establish a precise metrological index [27]. Thus, there is no consensus in most GBM in vivo studies for the best condition for MHT application, nor the reason for performing this therapy in multiple applications [13-15,22-26,28-30]. Variation in the number of MHT applications, its configurations, and the period for tumor evaluation are important aspects that need to be further explored. An additional important aspect of studies using MHT therapy in GBM models is the tumor induction out of brain region, this aspect of differents microenvironment is problematic for outcomes interpretations [31], and impair a correct translational MHT application.

MHT therapy outcome evaluation has been reported by different approaches using conventional techniques as follows: trypan blue [19,32,33], MTT [19,34-36], tunnel assay [32], live/dead assay [37,38], and Western blot [32] that in most cases have limitations for longitudinal analysis.

The therapeutic outcome evaluation by long periods is necessary due to tumor relapse cases. Imaging techniques are ideal for reducing the cost and animal numbers during the follow-up, and also providing dynamic structural, functional, and molecular information of the therapeutic process [39]. 
The imaging techniques most used are: bioluminescence (BLI) [40-43], near-infrared fluorescence (NIRF) [44], magnetic resonance imaging (MRI) [45,46], positron emission tomography (PET) [47-49], single photon emission computed tomography (SPECT) [50], magnetic particle imaging (MPI) [51], computed tomography (CT) [52], and behavioral assessment [53,54], which also has been indicate as tools for functional preclinical evaluations of longitudinal processes [40-43,47-49].

The bioluminescence technique has shown to be an excellent tool due to the best cost-effectivity for therapeutic outcomes evaluation in glioblastoma model [40-43], by having the advantage of high sensitivity with low background [55-57]. This technique involves the luciferin application to in vitro or in vivo models; luceferin reacts with luciferase enzyme, contained only in implanted tumor cells, this reaction is dependent of factors such as $\mathrm{Mg}^{2+}$, adenosinetriphosphate (ATP) and oxygen. The positive reaction offers linear correlation between luciferase concentration and photon emission, and can be detected and measured in real-time by the bioluminescence imaging (BLI) system [57].

BLI has some limitations regarding the two-dimensional (2D) nature of this imaging technique, which turns difficult the establishment of the brain depth in which the tumor is situated. In addition, BLI signal also depends on D-luciferin distribution, signal depth, and tissue absorption [44].

Another important technique for therapeutic follow up analysis in glioma models is the PET that display functional and biological information, such as metastatic lesion and tumor level staging of III and IV [49,58-62]. PET has high diagnostic, prognostic, and therapeutic evaluation value when integrating with CT or MRI informations [61,62] for drug metabolism and PET radiotracers as ${ }^{18} \mathrm{~F}$-2-fluoro-2-deoxy-D-glucose $\left({ }^{18} \mathrm{~F}-\mathrm{FDG}\right)$ which evaluates glucose metabolism. High-grade gliomas, such as GBM, are known to consume high glucose levels. This glucose consumption in humans is correlated with highly angiogenic tumors $[47,63]$, there is data available on this correlation in preclinical studies [64].

Preclinical studies usually report therapeutic progress evaluation by behavior assessment for long time follow up in different physiopathological conditions, but this information in antitumor therapy evaluation is scarce. Few studies evaluated spontaneous locomotion activity as a great potential for tumor progress analysis and the effects of different therapeutic modalities in liver tumor [65] and GBM models [54]. Recently, our group has developed a study on behavioral patterns regarding the progression of intracerebral C6-induced GBM model and its effect on locomotor movements [53], this last study was used as a comparative parameter for the therapy approached in the present study.

Therefore, the present study has the objective to evaluate the therapeutic effect of in vivo MHT technique in combination with SPION applied multiple times in brain tumor models. The best SAR value was previously determined in vitro using different combinations of frequency and magnetic field.

\section{Results}

\subsection{Evaluation of the SPIONa Specific Absortion Rate}

The SPIONa (100 nm) heating curves (time versus temperature) acquired through magnetic field of 100, 200, and 300 Gauss combined with oscillation frequencies of $309,364,420,557$, and $874 \mathrm{kHz}$ (Figure 1A-E) showed that none frequency combined with 100 Gauss were able to achieve the $43^{\circ} \mathrm{C}$ (therapeutic temperature). However, all frequencies combined with 200 and 300 Gauss fastly achieved the therapeutic temperature in less than $2 \mathrm{~min}$, showing an inverse relation between time and frequency to achieve the therapeutic temperature. The time for SPIONa reaches $43{ }^{\circ} \mathrm{C}$ after being submitted to each combination of the magnetic field and frequency is indicate in Table 1. 

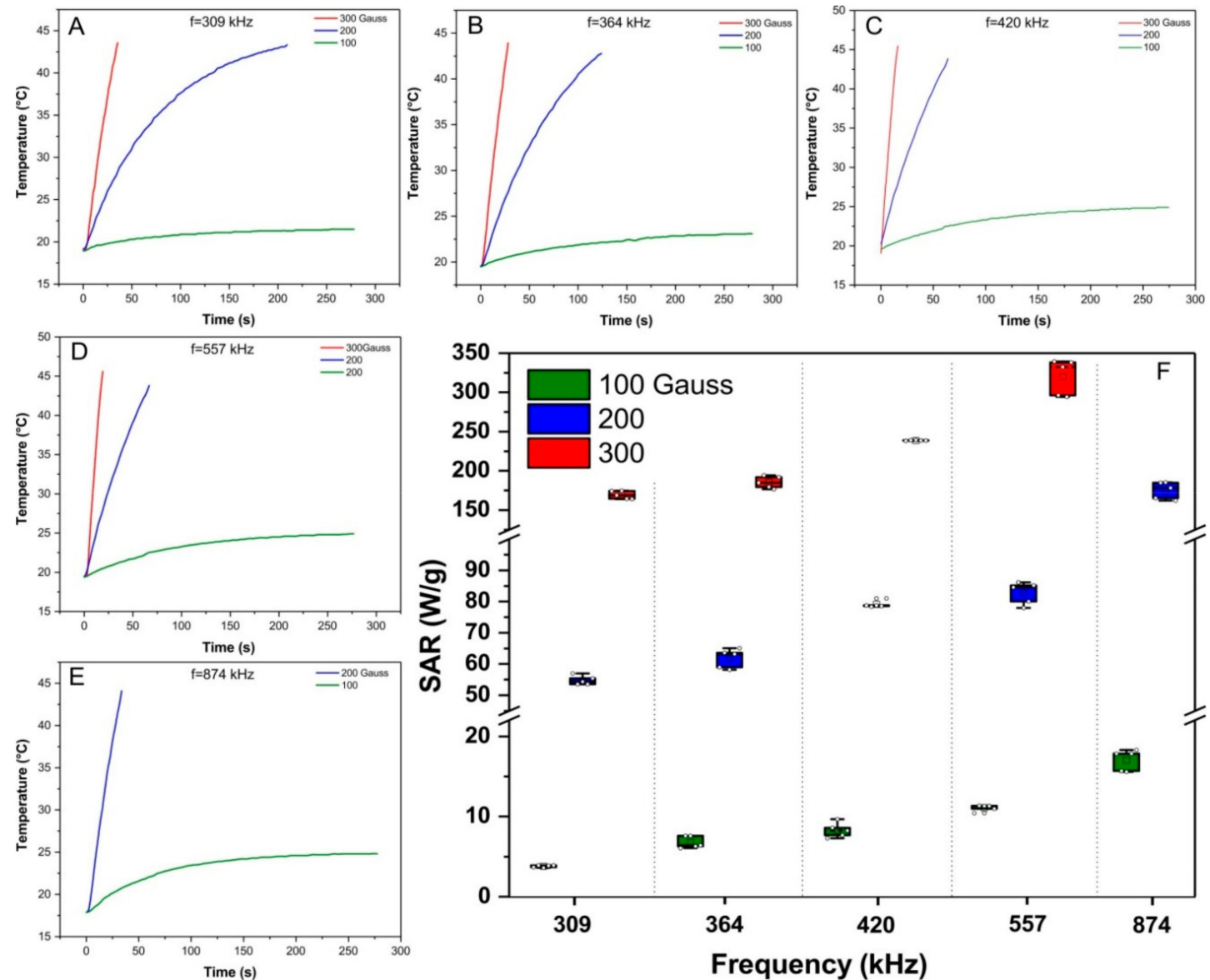

Figure 1. Evaluation of SPIONa heating potency exposed to the magnetic field of 100 (green line), 200 (blue line), and 300 Gauss (red line) combined with the oscillation frequencies of 309, 364, 420, 557, and $874 \mathrm{kHz}$. (A) Heating curves generated by SPIONa exposure submitted to alternating magnetic fields (AMF) with frequencies of $309 \mathrm{kHz}$; (B) $364 \mathrm{kHz}$; (C) $420 \mathrm{kHz}$ ); (D) $557 \mathrm{kHz}$; (E) $874 \mathrm{kHz}$. (F) Box plot graphic of SAR values calculated from the heating curves. SPIONa-aminosilane-coated superparamagnetic iron oxide nanoparticles.

Table 1. Heating time of SPIONa until achieving $43{ }^{\circ} \mathrm{C}$ (therapeutic temperature) as a function of magnetic field and oscillation frequency applied.

\begin{tabular}{|c|c|c|c|}
\hline Magnetic Field (Gauss) & Frequeny (kHz) & Mean Time to Achieve of $43^{\circ} \mathrm{C}$ (s) & $\mathbf{N}$ \\
\hline 300 & 557 & $14 \pm 2$ & 5 \\
\hline 300 & 420 & $15 \pm 3$ & 5 \\
\hline 300 & 364 & $23 \pm 4$ & 5 \\
\hline 300 & 309 & $26 \pm 4$ & 5 \\
\hline 200 & 874 & $32 \pm 5$ & 5 \\
\hline 200 & 557 & $57 \pm 4$ & 5 \\
\hline 200 & 420 & $58 \pm 4$ & 5 \\
\hline 200 & 364 & $104 \pm 5$ & 5 \\
\hline 200 & 309 & $119 \pm 7$ & 5 \\
\hline
\end{tabular}

$\mathrm{kHz}$ - kilohertz, SPIONa—superparamagnetic iron oxide nanoparticle coated with aminosilane.

SPIONa SAR values were obtained from heating curves, as shown in Figure 1F. The mean and standard deviation values of SPIONa SAR $(\mathrm{W} / \mathrm{g})$ are shown in Table 2 . ANOVA test showed significative difference in the comparison between the magnetic fields $(p<0.0 .001)$, oscillation frequencies $(p<0.001)$, as well as in the interaction between magnetic fields and oscillation frequencies $(p<0.001)$ 
Table 2. SPIONa SAR values for different combinations of magnetic field (100, 200, and 300 Gauss) and oscillation frequencie $(309,364,420,557$, and $874 \mathrm{kHz})$.

\begin{tabular}{ccccc}
\hline $\begin{array}{c}\text { Magnetic Field } \\
\text { (Gauss) }\end{array}$ & Frequency (kHz) & SAR Mean $(\mathbf{W} / \mathbf{g})$ & SD (W/g) & N \\
\hline 100 & 309 & 3.789 & 0.137 & 5 \\
& 364 & 6.785 & 0.736 & 5 \\
& 420 & 8.295 & 0.920 & 5 \\
& 557 & 11.078 & 0.403 & 5 \\
200 & 874 & 17.045 & 1.306 & 5 \\
& 309 & 54.757 & 1.460 & 5 \\
& 364 & 61.796 & 3.039 & 5 \\
& 420 & 79.117 & 1.101 & 5 \\
300 & 557 & 82.772 & 3.548 & 5 \\
& 874 & 175.112 & 10.897 & 5 \\
& 309 & 169.297 & 5.097 & 5 \\
& 364 & 185.464 & 7.646 & 5 \\
& 420 & 238.775 & 0.350 & 5 \\
& 557 & 320.070 & 22.818 & 5 \\
\hline
\end{tabular}

$\mathrm{kHz}$-kilohertz, SAR—specific absortion rate, SD—standard deviation.

Analyzing the different combinations of frequencies and magnetic field using the post hoc test (Table A1), we could verify that all frequencies oscillation tested combined with 100 Gauss magnetic field did not achieved significant differences in SAR value $(p>0.05)$.

The comparison of SAR value obtained with 309 and $364 \mathrm{kHz}$ of frequencies combined with 200 or 300 Gauss of the magnetic field also did not exhibited significant differences ( $p>0.05)$. Thus, only the SAR values obtained the combination between high frequencies $(420,557$ and $874 \mathrm{kHz})$ with 200 and 300 Gauss of magnetic field displayed significant differences $(p<0.001)$.

The highest SAR value was obtained by the combination of $557 \mathrm{kHz}$ oscillation frequency with 300 Gauss magnetic field and it was the parameter chosen for the in vitro studies. Considering the fact that high frequencies can cause undesirable electric currents [66], another parameter with high SAR but low-frequency values, such as a combination between $309 \mathrm{kHz}$ and 300 Gauss, was also chosen. Thus, two combinations of AMF were included for in vitro MHT evaluation in multiple applications: $557 \mathrm{kHz}, 300$ Gauss, and $309 \mathrm{kHz}, 300$ Gauss.

\subsection{In Vitro Study}

\subsubsection{Bioluminescence Signal Kinetics of C6 Cell Line Transduced with Luciferase}

To evaluate maximum intensity and sensitivity of BLI signal emitted by C6 cells transduced with luciferase was performed a kinetics analysis during $490 \mathrm{~min}$. The maximum intensity of BLI signal was obtained at $55 \mathrm{~min}$ after D-luciferin application followed by intensity reduction of signal. This pattern was observed in all cellular concentrations tested, showing high signal amplitude for high cellular concentrations. Thus, samples with $10^{6}, 10^{5}, 5 \times 10^{5}, 7.5 \times 10^{4}, 5 \times 10^{4}, 2.5 \times 10^{4}$ and $10^{4} \mathrm{C} 6$ cells showed the respective maximum intensities $(120.0 \pm 4.5) \times 10^{12},(59.4 \pm 4.05) \times 10^{10},(11.9 \pm 1.26)$ $\times 10^{10},(99.2 \pm 6.3) \times 10^{9},(73.1 \pm 7.0) \times 10^{9},(35.7 \pm 1.2) \times 10^{9}$ and $(11.7 \pm 1.4) \times 10^{9}$ photons $/$ sec, as shown in Figure 2. Images acquired in few time points were placed above the BLI curve to show the signal intensity changes according to the scale (Figure 2). The inserted figure (Figure 2) displays the curve amplification for lower cellular concentrations, in order to improve the details observed for different BLI intensities. 


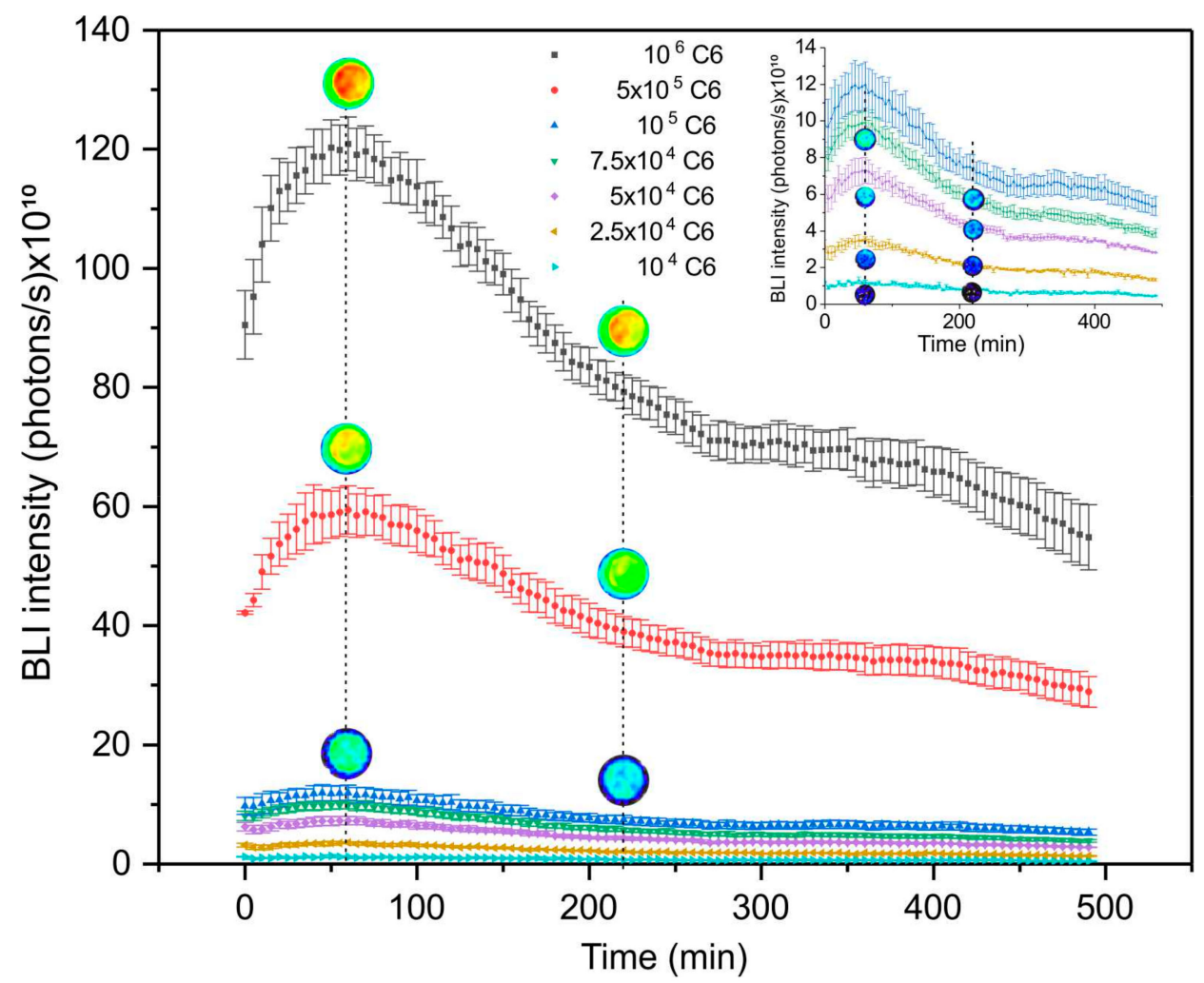

Figure 2. Bioluminescence kinetics signal as a function of $\mathrm{C} 6$ transduced with luciferase concentrations. The inset figure shows the curve amplification for lower cellular concentrations analysed for the variance between them.

\subsubsection{Evaluation of the SPIONa Internalization into C6 Cells}

The SPIONa internalization into C6 cells was detected through Prussian blue staining and Nuclear Fast red staining. Figure 3 shows the micrographs of the SPIONa internalization into C6 cells: using $100 \mu \mathrm{gFe} / \mathrm{mL}$ of SPIONa (Figure 3B,D) and $200 \mu \mathrm{gFe} / \mathrm{mL}$ of SPIONa (Figure 3F,H) and their respective controls (Figure 3A,C,E,G). SPIONa internalization was observed for either concentration tested. The highest SPIONa concentration $(200 \mu \mathrm{gFe} / \mathrm{mL})$ displayed a more intense internalization as shown in Figure $3 \mathrm{~F}, \mathrm{H}$, at $4 \times$ and $20 \times$ magnification, respectively.

\subsubsection{Evaluation of in Vitro MHT as a Function of Multiple Applications}

SAR values analyzed in item 2.1 displayed two sets of parameters for in vitro MHT application [(309 kHz_300 Gauss) and (557 kHz_300 Gauss)]. The effect of MHT evaluated by BLI signal, after each therapeutic application (Figure $4 \mathrm{~A}-\mathrm{C}$ ) showed similar behavior on cellular proliferation between experimental groups, in which the $\mathrm{C} 6$ cells were unlabeled with SPIONa [(C6); (C6-f1-B; C6-f2-B), as $\mathrm{f} 1=309 \mathrm{kHz}, \mathrm{f} 2=557 \mathrm{kHz}$ and $\mathrm{B}=300$ Gauss). The same occurred in C6_SPIONa group, in which the $\mathrm{C} 6$ cells were labeled with SPIONa, but were not exposed to AMF, showing that cell proliferation occurs indenpedent of SPIONa presence. These groups did not show significant differences for BLI signal $(p=1)$. However, groups exposed to SPIONa and AMF [(C6-f1-B with SPIONa); (C6-f2-B with SPIONa)] showed a reduction in BLI signal after MHT applications for both set of parameters compared to control. (Figure 4A-D). 


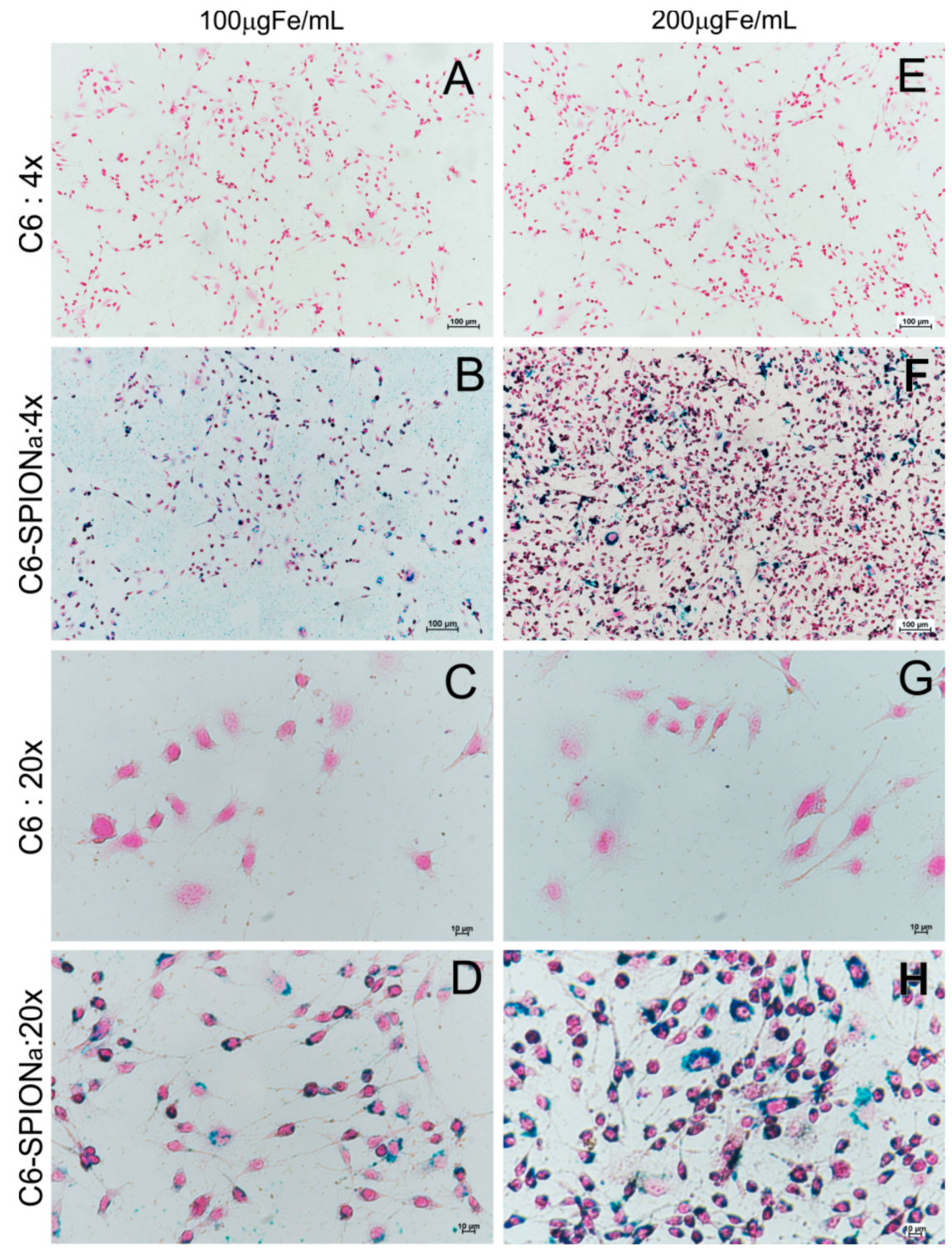

Figure 3. C6 cells labeled with SPIONa and stained with prussian blue and nuclear fast red. (A-D) Images of optical microscopy of the C6 cells labeled with $100 \mu \mathrm{gFe} / \mathrm{mL}$ of SPIONa concentration and respective controls and $(\mathbf{E}-\mathbf{H}) 200 \mu \mathrm{gFe} / \mathrm{mL}$ of SPIONa and respective controls $(4 \times$ and $20 \times$ magnification).

The BLI intenstities of each group were quantified, as shown in Table 3 and the results for statistic of repeated measuraments displayed significant differences for number of MHT applications $(p<0.001$, time effect), different experimental groups $(p<0.001)$ and interection between groups and number of MHT applications $(p<0.001)$. 


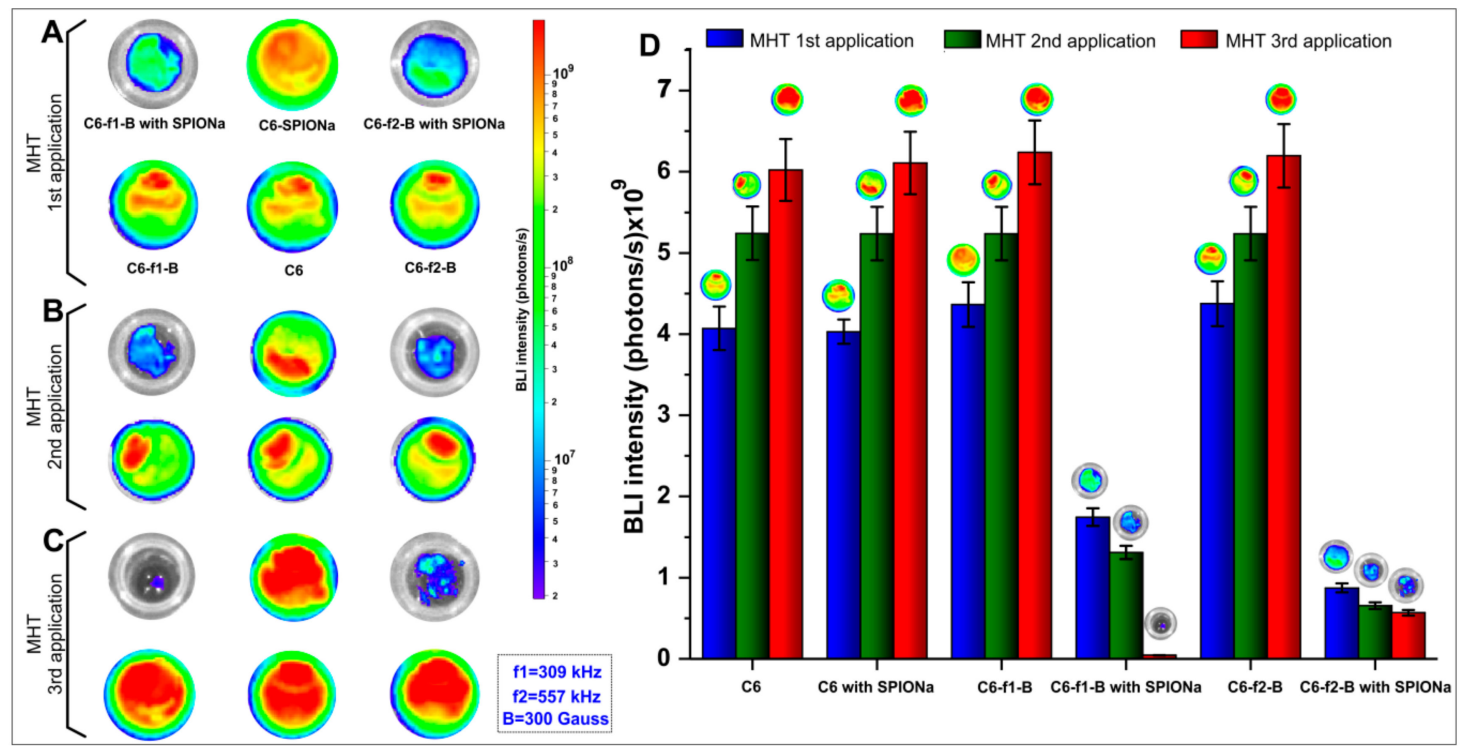

Figure 4. Evaluation of in vitro magnetic hyperthermia (MHT) therapeutic effect after multiple applications of bioluminescence imaging (BLI) signal: (A) BLI signal intensities after one MHT application, (B) BLI signal intensities after two MHT applications, (C) BLI signal intensities after three MHT applications, and (D) Histogram of BLI intensities in photons/s for each experimental group after one, two, and three MHT applications.

Table 3. BLI intensities values of samples submitted to in vitro MHT and their respective controls until three therapeutic applications.

\begin{tabular}{ccccc}
\hline $\begin{array}{c}\text { MHT } \\
\text { Applications }\end{array}$ & Groups & $\begin{array}{c}\text { Mean } \\
\text { (photons/s) }\end{array}$ & $\begin{array}{c}\text { SD } \\
\text { (photons/s) }\end{array}$ & $n$ \\
\hline One & C6 & $4.070 \times 10^{9}$ & $2.643 \times 10^{8}$ & 4 \\
& C6 with SPIONa & $4.030 \times 10^{9}$ & $1.479 \times 10^{8}$ & 4 \\
& C6-f1-B & $4.365 \times 10^{9}$ & $2.763 \times 10^{8}$ & 4 \\
& C6-f1-B with SPIONa & $1.748 \times 10^{9}$ & $1.124 \times 10^{8}$ & 4 \\
Two & C6-f2-B & $4.375 \times 10^{9}$ & $2.763 \times 10^{8}$ & 4 \\
& C6-f2-B with SPIONa & $8.730 \times 10^{8}$ & $5.501 \times 10^{7}$ & 4 \\
& C6 & $5.242 \times 10^{9}$ & $3.301 \times 10^{8}$ & 4 \\
& C6-SPIONa & $5.237 \times 10^{9}$ & $3.297 \times 10^{8}$ & 4 \\
& C6-f1-B & $5.237 \times 10^{9}$ & $3.297 \times 10^{8}$ & 4 \\
Three & C6-f1-B with SPIONa & $1.309 \times 10^{9}$ & $8.243 \times 10^{7}$ & 4 \\
& C6-f2-B & $5.237 \times 10^{9}$ & $3.297 \times 10^{8}$ & 4 \\
& C6-f2-B with SPIONa & $6.546 \times 10^{8}$ & $4.121 \times 10^{7}$ & 4 \\
& C6 & $6.022 \times 10^{9}$ & $3.792 \times 10^{8}$ & 4 \\
& C6-SPIONa & $6.110 \times 10^{9}$ & $3.847 \times 10^{8}$ & 4 \\
& C6-f1-B & $6.241 \times 10^{9}$ & $3.929 \times 10^{8}$ & 4 \\
& C6-f1-B with SPIONa & $4.364 \times 10^{7}$ & $2.748 \times 10^{6}$ & 4 \\
& C6-f2-B & $6.197 \times 10^{9}$ & $3.902 \times 10^{8}$ & 4 \\
& C6-f2-B with SPIONa & $5.673 \times 10^{8}$ & $3.57 \times 10^{7}$ & 4
\end{tabular}

MHT—Magnetic hyperthermia, SD—standard deviation.

Analyzing the therapeutic group with AMF presence and SPIONa internalization, a significant reduction was observed in BLI signal intensity compared to their respective controls $(p<0.001$, red color), as shown in Figure 5. 

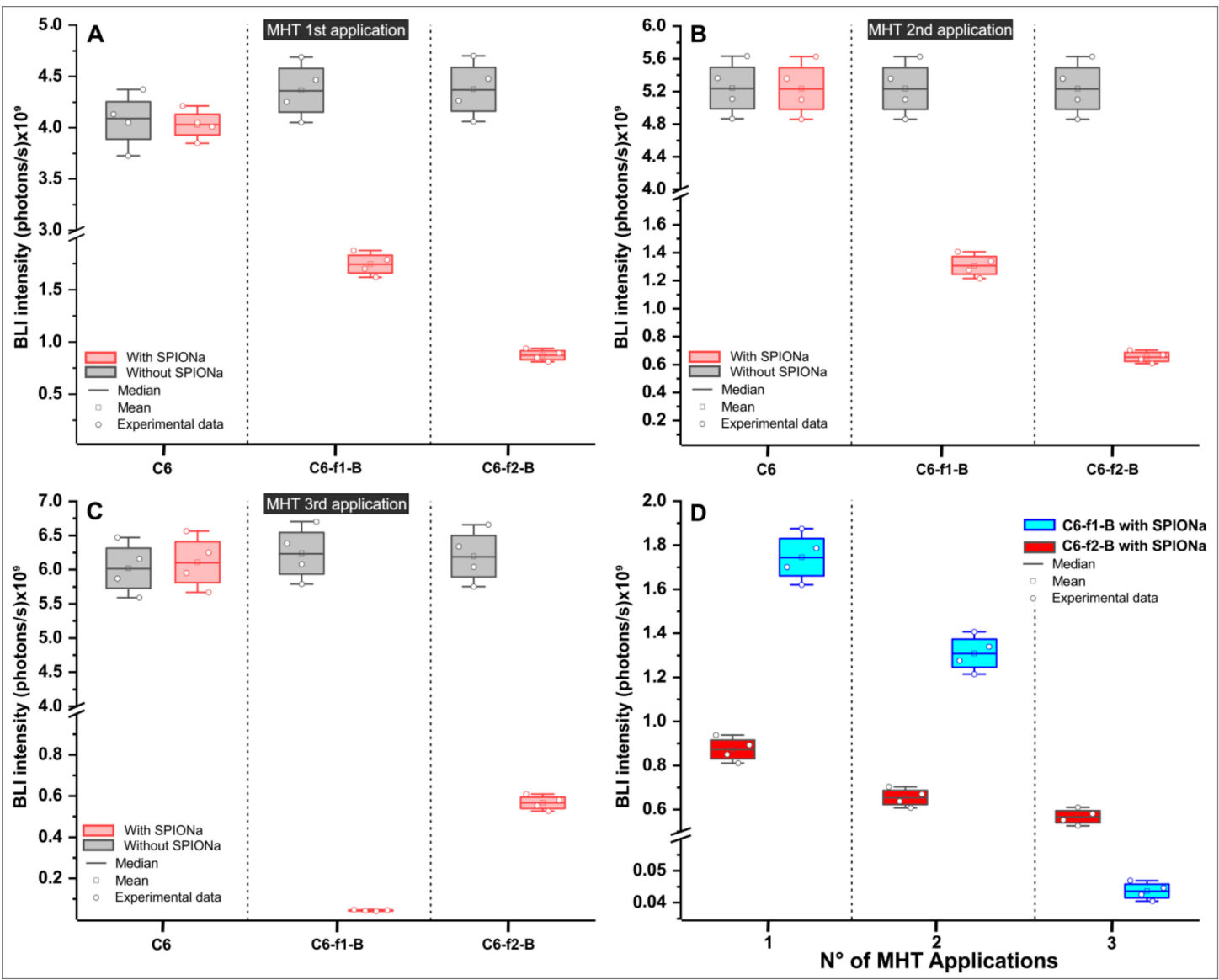

Figure 5. Comparison of BLI signal intensity between groups with and without SPIONa in the MHT multiple applications. The box plot shows the BLI intensity in the experimental groups (A) after one MHT application, (B) after two MHT applications, (C) after three MHT applications, and (D) the comparison of BLI intensities between the therapeutic groups, analyzing the two sets of AMF configuration: (f1-B e f2-B f1 = $309 \mathrm{kHZ}$; $\mathrm{f} 2=557 \mathrm{kHz}$ e B = 300 Gauss).

After one MHT application, the C6-f1-B group that used frequency of $309 \mathrm{kHz}$ showed significant BLI intensity reduction, decreasing from $(4.365 \pm 0.276) \times 10^{9}$ to $(1.748 \pm 0.112) \times 10^{9}$ photons/s $(p<0.001$, Appendix A-Table A2), comparing cells without SPIONa and with SPIONa, respectively.

The C6-f2-B group that used $557 \mathrm{kHz}$ of frequency also showed a significant reduction of the BLI intensity from $(4.367 \pm 0.276) \times 10^{9}$ to $(8.730 \pm 0.873) \times 10^{8}$ photons $/ \mathrm{s}(p<0.001$, Appendix A-Table A2), comparing cells without SPIONa and with SPIONa, respectively.

In addition, comparing the BLI intensities between C6-f1-B group and C6-f2-B group both with SPION, the highest value of frenquency (C6-f2-B group) showed a significant reduction of BLI intensity in comparison with C6-f1-B group that used low frenquecy values $(p<0.001$, Appendix A-Table A2).

After two MHT applications (the second application occurred three days after the first MHT application), as shown in Figure 5B, the BLI intensities had similar pattern of reduction described for the first MHT applicaton. Thus, the C6-f1-B group ( $309 \mathrm{kHz}$ of frequency) showed significant reduction of the BLI intensity decreasing from $(5.237 \pm 0.329) \times 10^{9}$ to $(1.309 \pm 0.824) \times 10^{9}$ photons $/ \mathrm{s}(p<0.001$, Appendix A-Table A3), comparing cells without SPIONa and with SPIONa, respectively.

The C6-f2-B group that used $557 \mathrm{kHz}$ of frequency also showed a significant reduction of BLI intensity decreasing from $(5.237 \pm 0.329) \times 10^{9}$ to $(6.546 \pm 0.412) \times 10^{8}$ photons $/ \mathrm{s}(p<0.001$, Appendix A-Table A3), comparing cells without SPIONa and with SPIONa. In addition, comparing the BLI intensities between C6-f1-B group and C6-f2-B group both with SPIONa, the highest value of 
frenquency (C6-f2-B group) showed a significant reduction of BLI intensity compared to C6-f1-B group that used low frenquecy value ( $p=0.047$, Appendix A-Table A3).

After submitted to three MHT applications (the third application occurred three days after the second MHT application), the BLI intensity results displayed a different pattern from others MHT applications (Figure 5C). In this case, the BLI intensity had a superior decay after submitted to AMF with low frequency value (C6-f1-B group with SPIONa, with $309 \mathrm{kHz}$ ), comapred with the group that used the high frequency value (C6-f2-B group with SPIONa, with $557 \mathrm{kHz}$ ). Thus, the BLI signal intensity decreased from $(6.241 \pm 0.392) \times 10^{9}$ to $(4.364 \pm 0.274) \times 10^{7}$ photons/s in the C6-f1-B group and from $(6.197 \pm 0.390) \times 10^{9}$ to $(5.673 \pm 0.357) \times 10^{8}$ photons/s in the C6-f2-B group.

Consequently, the BLI intensities showed significant difference between C6-f1-B and C6-f2-B groups with SPIONa in the first MHT application $(p<0.001$; Appendix A-Table A3), in the second MHT application ( $p=0.047)$, but not in the third MHT application ( $p=0.463$; Appendix A-Table A4). The MHT therapeutic effect evaluated by BLI intentisty showed that the C6-f1-B with SPIONa group had a more effective therapeutic result than C6-f2-B with SPIONa group, but without significative difference.

Figure 5D display significant difference in C6-f2-B with SPIONa group between the first and second MHT application $(p<0.001)$, but not between the second and third MHT application $(p=0.066)$. However, in C6-f1-B with SPIONa group over time analysis of BLI intensity showed significant differents between all over time comparisons, the first with second and second with third MHT applications $(p<0.001)$. Due to the pattern of evolution occurred over time in the C6- $\mathrm{f} 1-\mathrm{B}$ with the SPIONa group and the low risk of the generation of parasitic electric currents with the low value of frequency, the combination of AMF and frequency to be applied in vivo MHT process was $309 \mathrm{kHz}$ and 300 Gauss.

Thus, the in vitro MHT analysis in multiple applications allowed us to choose the more effective parameters of AMF to apply into in vivo analysis, considering the high SAR value, better efficacy for multiple therapy applications and using lower oscillation frequencies and magnetic field.

\subsection{In Vivo Study}

\subsubsection{Evaluation of Tumoral Growth by Histological Analysis}

The volumetric and morphological analysis of C6 cells implanted in the motor cortex using robotic stereotaxic was performed by histology using H\&E staining. In the sham group was observed only the effect of Dulbecco's Modified Eagle's Medium: nutrient mixture F-12 (DMEM/F12) implanted (Figure 6A,B). After seven days of tumor induction, in the left hemisphere was observed the tumor development (Figure 6C,D), showing the migratory tendency to callosum corpus, more clearly observed at the 14th day (Figure 6E,F), at the 21st day the tumor already compresses the corpus callosum and achieve the ventricle ipsilateral. The tumor evaluation at 28th day, display a big tumor mass compressing the ventricle and exceeding their limits for the other hemisphere, this tumor is inadequate for MHT multiple applications due to animal short survival of 18 days 


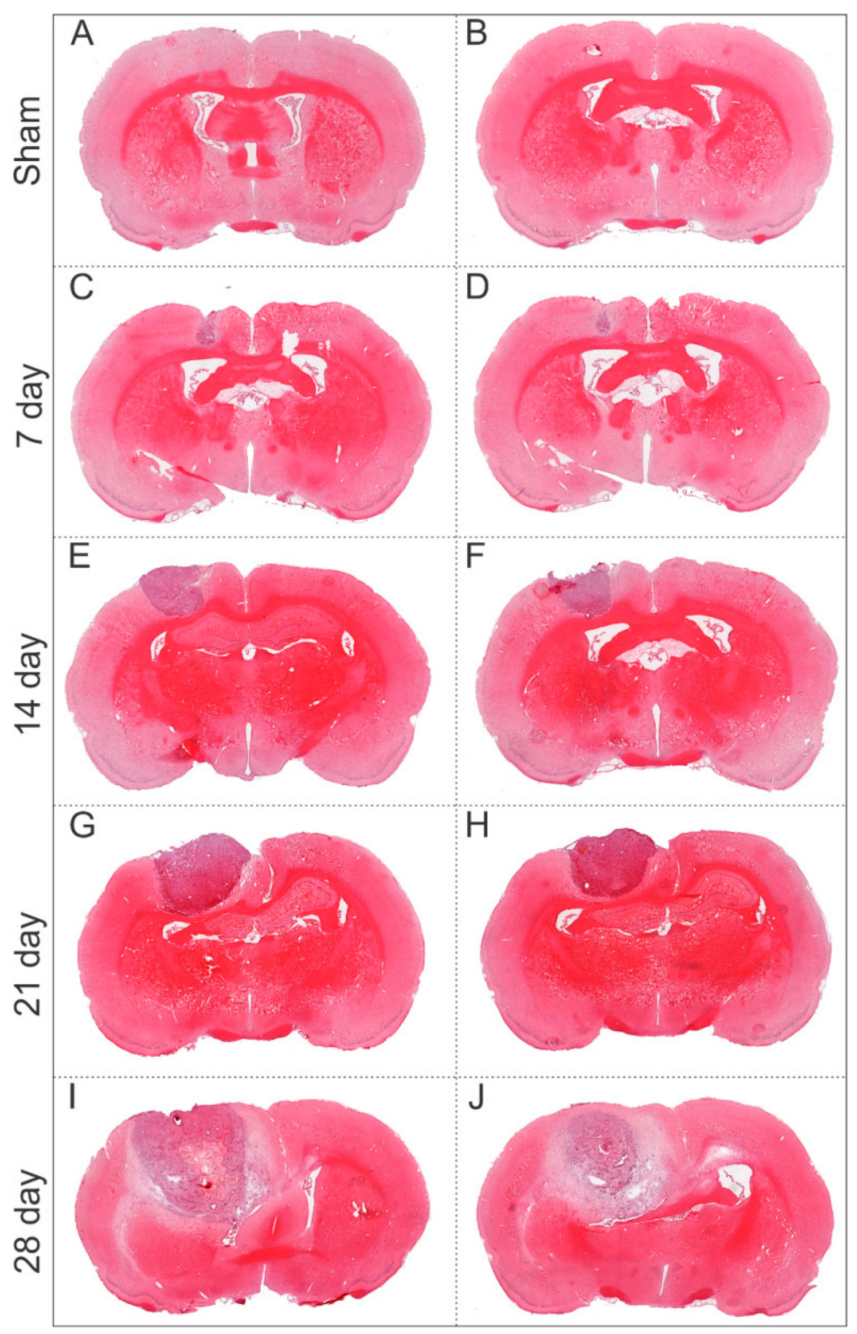

Figure 6. Evaluation of tumor growth by histology using H\&E over 28 days after C6 cells tumor induction. (A,B) Sham group, brain without tumor induction, only medium injection. (C,D) Histological sections of tumoral tissue after seven days of tumor induction, $(\mathbf{E}, \mathbf{F})$ after 14 days, $(\mathbf{G}, \mathbf{H})$ after 21 days, and $(\mathbf{I}, \mathbf{J})$ after 28 days.

\subsubsection{Evaluation of MHT Multiple Applications in Vivo}

After in vitro MHT evaluation was possible to choose the following set of parameters AMF, 300 Gauss of the magnetic field and $309 \mathrm{kHz}$ of frequency oscillation. The MHT multiple applications were performed at the 14th, 17th and 21st days after tumor induction for the first, second, and third MHT applications, respectively. BLI images of all experimental groups were obtained at 13 th day as the baseline measurement before the first MHT application, at 22nd day, two days after multiples MHT applications as an acute measure of therapeutic effect of MHT and at 32nd day, 12 days after multiples MHT applications, as late measurement of therapeutic effect of MHT (Figure 7A,B).

Comparing the BLI signal intensities of each experimental group, the sham group did not show any BLI signal after D-luciferin application (Figure 7A-first line of images, and Figure 7B-black color bars). The tumor group that did not receive the MHT treatment, showed significant increase in the BLI signal intensity over time ( $p<0.05$, time effect; gray bars of Figure $7 \mathrm{~B}$ ), $68 \%$ and $274 \%$ of tumor growth, comparing the BLI intensity at 22nd and 32nd with the baseline (13th day). 


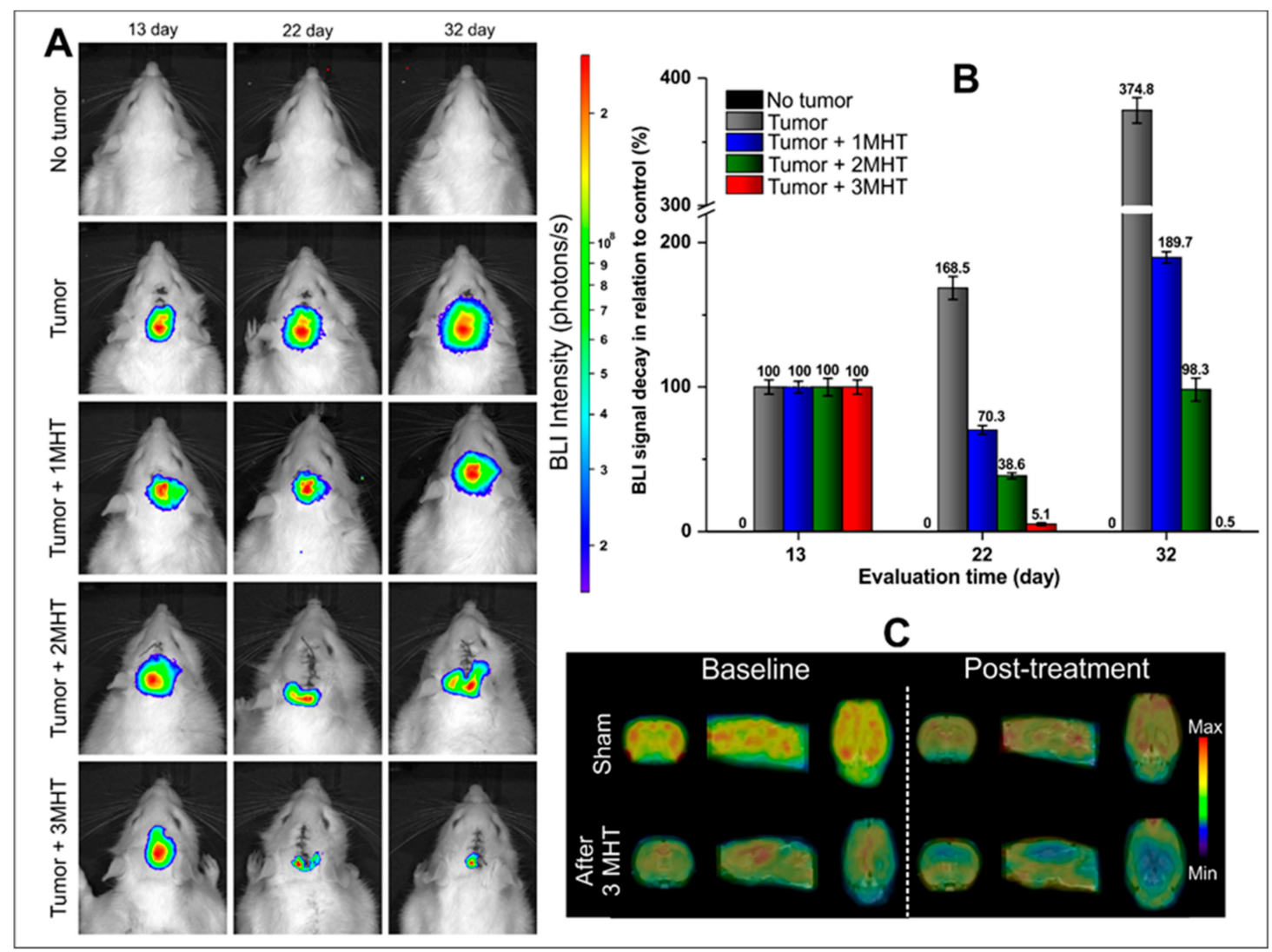

Figure 7. Evaluation of the MHT therapeutic process in multiple applications by BLI and ${ }^{18}$ F-FDG-PET. (A) BLI images of experimental groups at 13th (baseline), 22nd, and 32nd day after tumor induction, BLI signal changes according to the number of MHT applications. (B) Evaluation of BLI signals percentual after multiple applications of MHT therapeutic process. (C) Illustrative ${ }^{18}$ F-FDG-PET images fused to MRI templates of animals not submitted to tumoral induction (sham), animals submitted to tumor induction after three MHT applications, before (baseline) and post-treatment through ${ }^{18}$ F-FDG-PET.

The groups that received MHT therapy showed reduction of BLI signal proportional to the number of MHT applications, refleting in the tumoral size visualized in BLI signal at 22nd day (Figure 7A- B blue, green and red bars). The animal's tumor groups that received one, two and three applications showed reduction of BLI signal-29.7\%,61.4\%, and $94.9 \%$ related to the reduction of tumor size in comparison to the baseline, with significant difference between groups and over time $(p<0.001)$. However, the decay of tumor signal was not constant, since on 32nd day of evaluation, groups with one and two MHT applications, showed the respective increase of $119.6 \%$ and $59.7 \%$ in the BLI signal compared to 22nd day of measurement, and significant differences between groups $(p<0.001)$.

The tumor group in which MHT therapy was applied three times showed at 32nd day absense of tumor relapse, mantaining the decay of BLI signal during late evaluation. The over time comparison of this group showed significant differences between 32nd day measures with the baseline $(p<0.001)$. Thus, three MHT applications revealed to be an effective therapy with almost complete tumoral mass elimination. These results were ilustrated by ${ }^{18} \mathrm{~F}$-2-fluoro-2-deoxy-D-glucose $\left({ }^{18} \mathrm{~F}-\mathrm{FDG}\right) \mathrm{PET}$ images (Figure 7C), in which the glucose metabolism was analyzed before (baseline) and after the three MHT applications, comparing the sham and Tumor+3MHT group. In the sham group, the ${ }^{18}$ F-FDG uptake was constant, comparing the time points. Instead, the Tumor+3MHT group presented important decrease in glucose metabolism $\left({ }^{18} \mathrm{~F}-\mathrm{FDG}\right.$ uptake) compared to the baseline. 


\subsubsection{Spontaneous Locomotion Evaluation in Multiple MHT Applications}

The spontaneus locomotor activity was analyzed in the experimental groups of MHT multiple applications using four behavior parameters: slow horizontal movement (S-MOV), fast horizontal movement (F-MOV), slow rearing (S-REA), and fast rearing (F-REA) at 0, 7, 14, 19, 24, and 32 days after tumor induction, as shown in Figure 8.

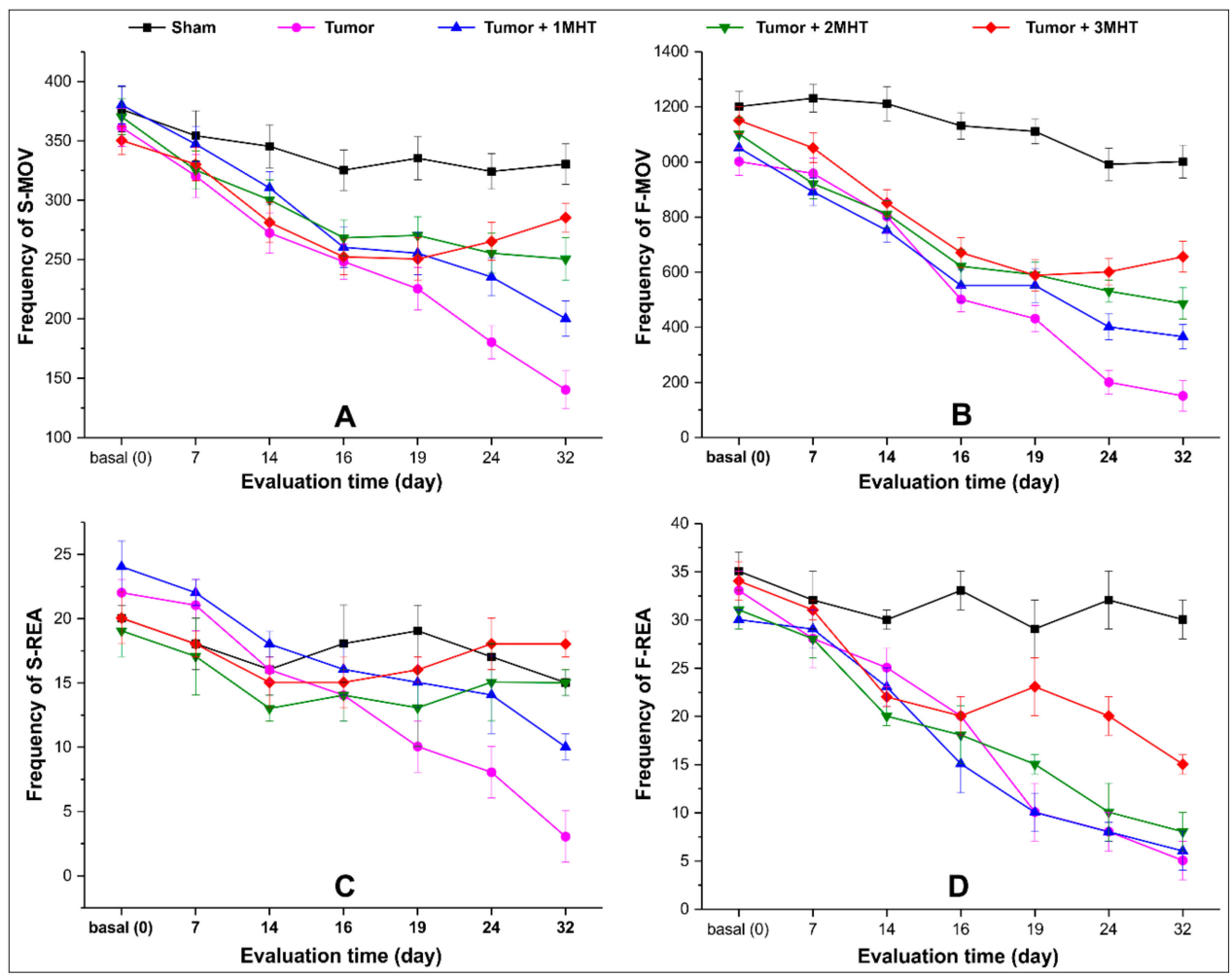

Figure 8. Spontaneus locomotor activity evaluation during multiples MHT applications. (A) Slow horizontal moviment (S-MOV), (B) fast horizontal moviment (F-MOV), (C) slow rearing (S-REA), and (D) fast rearing (F-REA), evaluted at $0,7,14,16,19,24$, and 32 day.

Basal measurament for all groups did not show significant differences in any parameter of the behavioral evaluation $(p>0.05)$. At 7 and 14 days (Figure 8A-D), before the first MHT application the groups with tumor induction showed a significant reduction of movement frequency $(p<0.05)$, with exception in F-MOV parameter for the sham group (without a tumor) that show significant difference compared to other groups submitted to tumor induction $(p<0.001$, Figure $8 \mathrm{~B})$

In the acute MHT evaluation (at 14, 16, and 19 days after tumor induction), the frequency of S-MOV, F-MOV, S-REA, and F-REA showed a soft improvement after MHT, but was not durable for the tumor groups that received only one or two MHT applications. The late MHT evaluation of these groups (at 24th and 32nd days after tumor induction), the frequency of all parameter of movement became regressed, showing a significant difference between groups $(p<0.001)$. However, the tumor group that received three MHT applications after 19 days of tumor induction had a significant improvement in all parameters, mainly in horizontal movements (S-MOV and F-MOV) compared to the others tumor groups that received one or two MHT applications $(p<0.001)$, keeping this pattern constant until 32nd day.

Thus, the behavior assessment through spontaneous locomotor activity showed a decay of movement frequency in all parameters analyzed for all groups. The frequency of S-REA decayed until 14 days and after occurred small variation for tumor group with MHT therapy and sham group. For other parameters, only the late MHT evaluation was more decisive for discrimination between groups. 


\section{Discussion}

The present study demonstrated the efficacy of MHT multiple applications for glioblastoma tumor model based on structural and functional evaluation, reaching functional improvement until the latest stages of evaluation. Thus, for this success, many paramenters of MHT therapy were evaluated and had important impact in the achievement of these results.

Characteristics as size, shape, coating, colloidal stability, surface charge, and magnetic properties influence the SPION interaction with tumor tissue and consequently with the MHT process $[67,68]$. In a previous study developed by our group, we have developed data on stability and hydrodynamic diameter $\left(\mathrm{D}_{\mathrm{H}}\right)$ distribution of SPIONa dispersed in aqueous solution. These data were generated by dynamic light scattering and demonstrated stability during $24 \mathrm{~h}$ of evaluation, without forming agglomerations, mantaining the $100 \mathrm{~nm}$ of $\mathrm{D}_{\mathrm{H}}$ [69]. Similar results were reported by other groups that compared SPIONa with uncoated SPION disperse in aqueous solution and their stability. These studies have also shown that SPIONa do not display agglomeration [70], in agreement with the SPION coating influence on their stability and heating features [10].

Another SPION characteristic used in the MHT technique is the high SAR that depend on size, shape, composition, magnetization, magnetic interaction, and concentration of SPION, also as the frequency and strength of magnetic field applied [71], it is necessary to avoid magnetic field with the high oscillation frequencies due to heating by eddy current [72]. Therefore, in our study, a set of frequencies and magnetic fields were evaluated for the obtainment of SPIONa heating curves and posterior SAR values as shown in Table 2, hanging from $3.789 \mathrm{~W} / \mathrm{g}$ (100 Gauss; $309 \mathrm{kHz}$ ) to the maximum value of $320.07 \mathrm{~W} / \mathrm{g}$ ( 300 Gauss; $557 \mathrm{kHz}$ ).

Similarly, SPIONa SAR value was reported by Yuan et al. [73], using an AMF with $9.12 \mathrm{kA} / \mathrm{m}(114$ Gauss) and $250 \mathrm{kHz}$ of oscillation frequency to determine the SAR value of $14.96 \mathrm{~W} / \mathrm{g}$. The variability of AMF parameters to obtained the SPIONa SAR values turns difficulty the comparison between studies, but the similarity of the SPIONa heating capacity can be performed using the intrinsic loss power (ILP $\left.=\mathrm{SAR} /\left(\mathrm{fxB}^{2}\right)\right)$ which results in the SPION proprieties excluding the influence of AMF and oscillation frequency [74]. In our study, the ILP was the $\sim 0.680 \times 10^{-8} \mathrm{Wg}^{-1} \mathrm{Oe}^{-2} \mathrm{~Hz}^{-1}$, acceptable value when compared to ILP value defined by Yuan et al. [73] study, calculated in $0.46 \times 10^{-8} \mathrm{Wg}^{-1} \mathrm{Oe}^{-2} \mathrm{~Hz}^{-1}$, considering the inhomogeneities of the magnetic field.

A SPION used in clinical applications [16], coated with dextran was submitted to AMF $(11 \mathrm{kA} / \mathrm{m}$ (137 Gauss; $150 \mathrm{kHz}$ ) and showed SAR value of $286 \mathrm{~W} / \mathrm{g}$ and correspondent ILP value of $0.100 \times 10^{-8}$ $\mathrm{Wg}^{-1} \mathrm{Oe}^{-2} \mathrm{~Hz}^{-1}$. Other SPION type with similar features and applications, but coated with starch, also was characterized and showed the respective values of SAR and ILP; $230 \mathrm{~W} / \mathrm{g}$ and $1.4 \times 10^{-8}$ $\mathrm{Wg}^{-1} \mathrm{Oe}^{-2} \mathrm{~Hz}^{-1}$ [75]. However, the ILP value produced by computational simulations for this SPIONa was $1.02 \times 10^{-8} \mathrm{Wg}-1 \mathrm{Oe}-2 \mathrm{~Hz}-1$. Thus, it is possible based on the previuos data to state that SPION coated with dextran has no potential for MHT, instead SPION coated with starch has potential for MHT [73]. Analyzing these results, we can affirm that the SPION coated with dextran used in the Ribas et al. study [16], have no potential for MHT, compared to the SPION coated with starch used in the Ludwig et al. study [75], and futher comparing the ILP values of SPION starch coated with SPIONa we can conclude that both have equal potential for use in MHT application. The difference in ILP values is attributed to the non-homogeneous magnetic field generated by the coil and the coating material used in the SPION [73,75].

On the other hand, an important aspect of MHT process is the SPION internalization for the cells into tumoral tissue, the surface charge (zeta potential) of SPION has influence in the pathways of SPION internalization by tumoral cells [67] due to the negative character of the lipidic bilayer found in the cell membrane external surface [76]. The positive character of SPION surface contributes favorably for their internalization process due to electrostatic action, as seen in C6 cells [77-80]. In our study, the SPIONa zeta potential displayed positive character $(+20 \mathrm{mV})$ which favored the nanoparticle internalization into $\mathrm{C} 6$ tumoral cells as observed in our results. The SPIONa internalization was greater according to higher concentrations values, without any evidence of toxicity. This type of strategy was 
reported in other studies that used SPION coated with polyethyleneimine (PEI) with a positive surface charge of $+29.28 \mathrm{mV}$, promoting their internalization in macrophages cells as much as in tumoral cells compared to SPION with zeta potential of $-0.52 \mathrm{mV}$ [11]. In addition, a comparative study of SPION internalization and toxicity showed good internalization rate and low toxicity for aminosilane-coated SPION with positive surface charge [12].

After the nanoparticles heating power evaluation through SAR and SPIONa internalization into C6 cells, was performed the in vitro study with MHT multiple applications. The BLI signal evaluation after the first and second MHT applications showed that the C6 cells viability was affected proportionaly to the applied frequency value. So, the cellular viability using AMF with high frequency $(557 \mathrm{kHz}$; 300 Gauss), was reduced to $20.02 \%$ after one MHT and $12.49 \%$ after two MHT applications, for AMF low frequency ( $309 \mathrm{kHz} ; 300$ Gauss), the viability was reduced to $40.03 \%$ and $25.02 \%$ after one and two MHT application, respectively.

In the third MHT application, the viability was inversely proportional to the frequency applied; the low and high frequency of AMF used showed the respective cellular viability reduction of $0.7 \%$ and $9.15 \%$. Thus, in the third MHT application, the low efficiency of the AMF high frequency can be connected to a possible intracellular mechanism of heat stress resistance, likely related to heat shock proteins, such as hsp70 [13].

Several studies using in vitro MHT demonstrated different approaches and results, such as a study using SPION coated with aminolisane $(50 \mathrm{~nm})$ submitted to AMF with $220 \mathrm{kHz}$ and 139.2 ampere (4.0 $\times 4.3 \mathrm{~cm}$ diameter coil) for $2 \mathrm{~h}$ showed $50 \%$ efficiency $24 \mathrm{~h}$ after finished the therapy. In this study, the SPION zeta potential was $-10 \mathrm{mV}$, a value that might not favor the SPION internalization by cells [81]. Another study that synthesized aminosilane-coated SPION, they were submitted to low frequency ( $1.16 \mathrm{uT}$ and $350 \mathrm{kHz}$ ) and a single MHT application for $30 \mathrm{~min}$, showing 30\% of K7M2 cells viability by BLI $24 \mathrm{~h}$ after finished the therapy [34], and others studies that used single MHT therapy application displayed efficiency superior than 50\% [32,35].

Another studies in vitro using U-87MG cells and employing multiple MHT applications, used SPION with $106.2 \mathrm{~nm}$ of diameter submitted to AMF (750 kHz; 200 Gauss), and four days of MHT applications ( 1 per day during $2 \mathrm{~h}$ ) showing 50\% efficiency after the first MHT application and $80 \%$ after three days indicating the importance of latency period for MHT therapeutic efficacy analysis [30]. The precaution adopted in our study of two days latency for MHT efficiency analysis, was very important, because during this latency period it is possible the occurrence of cell death by membrane permeabilization or rupture, increased reactive oxygen species or heat shock protein (Hsp) expression [32]. Others studies that used two MHT applications did not showed significant differences in efficiency between first and second MHT applications, their evaluations occured right after the last MHT application within 30 min between applications [28,29].

Fractionated use of radiotherapy (or multiple applications) improves overall survival in patients, however, even though it has been applied for decades, there is still no optimal dose fraction established, although evidences suggest that moderately hypofractionated radiotherapy causes a survival increasing from 12 to 16 months [82,83]. Similar to radiotherapy, there is still no optimal dose fraction established for MHT therapy, and further studies are necessary to establish the best AMF parameters, periodicity of application, SPION dose and fractionation of MHT application. Although the MHT is already being applied in GBM clinical trials phase I and II as adjunctive therapy, there is few reports since 2009 [18, 20,21,84], from in vivo preclinical studies supporting MHT fractional doses applications [14,15,22-26]. A source of variability concerns to the site of GBM tumor induction and many of these models are not induce intracerebrally but subcutaneously in non-brain regions, which may difficult later translation of the technique [13-15,25]. Though, this is changing, and more recently, studies have been developing intracerebral models of GBM with various cell lines $[22,23,26]$. Our study is the first to evaluate the effects of multiple MHT applications using C6 cells in an intracerebral model of GMB.

MHT therapy with multiple applications in vivo has been reported in literature with several diferent times of applications such as: 2 [85], 3 [13-15], 4 [25], 7 [26], 15 [24], 24 [23], and 27 [22]. Studies 
for MHT with multiple applications in non-cerebral glioma model $[14,15]$ used the $11^{\text {th }}$ day after T9 cell induction, to initiate the MHT using the magnetite cationic liposomes (MCL) submitted to $118 \mathrm{kHz}$ and $30.6 \mathrm{kA} / \mathrm{m}$ ( $384 \mathrm{Oe}$ ) for $30 \mathrm{~min}$ per session, with $24 \mathrm{~h}$ of intervals between 3 applications. These three applications resulted in the respective tumor reduction of $20 \%, 60 \%$, and $87.5-88.9 \%$, and the animals survival was evaluated up to 30 days after applications [15].

Studies involving brain tumor models (ALTS1C1 cells) that used oleic acid-coated SPION submitted to four MHT applications for $12 \mathrm{~min}$ at $37 \mathrm{kHz}$ and $2.5 \mathrm{kA} / \mathrm{m}$, increased the average survival from 11 (control) to 31 days [25]. Another study that used SPION submitted to AMF with $200 \mathrm{kHz}$ and 300 Gauss and SAR value of $57 \mathrm{~W} / \mathrm{g}$ in 15 MHT applications showed therapeutic success after 28 days of tumor induction with initial tumor size of $3 \mathrm{~mm}^{3}$ [24]. These results differ from ours, which used SAR value of $169.279 \mathrm{~W} / \mathrm{g}$ when submitted to $309 \mathrm{kHz}$ and 300 Gauss of AMF, resulting in the tumor reduction after three applications of MHT and enhanced survival compared to the control group.

For the comparative analysis between studies, it is important to consider the tumor volume and the concentrations of SPION administered into tumor tissue [22-24]. There is evidence that the same MHT parameters applied in different tumor sizes showed diverse results, tumor size of $3 \mathrm{~mm}^{3}$ disappear completely, diferently from tumor size of more than $25 \mathrm{~mm}^{3}$ that showed tumor regression in varied proportions [24]. A study with three MHT application (SAR $=52 \mathrm{~W} / \mathrm{g}$ ) per week over 16 days, with session lasting $30 \mathrm{~min}$, was also unable to completely destroy the tumor mass due to tumor size $\left(100 \mathrm{~mm}^{3}\right)$, not preventing tumor recurrence $[22,23]$. The same did not occur in tumors with size between $1.5-2 \mathrm{~mm}^{3}$, displaying the importance of tumor size evaluation for proper therapy planning, which was done in the present study, the application of MHT started with similar tumor volumes.

Longitudinal follow-up of both tumor growth and therapeutic process can be performed by the BLI technique. Our study used this technique to verify the treatment efficiency during the acute phase to verify the effectiveness of therapy and its relation to the number of MHT application and in the late phase after treatment aiming to verify the tumor recurrence. However, one and two MHT applications were not suffient to prevent tumor recurrence in the late phase of evaluation, 32 days after tumor induction, which did not occured when three MHT sessions were applied. This last approach maintained the therapeutic efficacy observed in the acute evaluation.

Some other studies also used BLI technique to evaluate the MHT therapy efficiency. Chen et al. [26], evaluated the MHT therapeutic effect at 7, 14, 21, and 28 days after tumor induction, starting the therapy four days after tumor induction and having it applied for seven consecutive days for $1 \mathrm{~h}$ using the AMF parameters of 1 Tesla and $20 \mathrm{~Hz}$. This study showed that only $60 \%$ of animals displayed low BLI signal during acute phase and during the late phase, the $40 \%$ animal with no BLI signal sustained the same condition with no tumor recurrence. Alphandèry et al. [22] evaluated MHT therapy during 56 days, applying $27 \mathrm{MHT}$ sections $(202 \mathrm{kHz} ; 27 \mathrm{mT})$ evaluating the therapeutic efficacy one day after each session and until 150 days (late stage), 100\% of animals had no BLI signal 68 days after tumor induction without tumor recurrence at the late stages of evaluation (150 days after tumor induction).

In addition to BLI, another imaging technique used for longitudinal studies is PET, which analyzes the decreasing in glucose metabolism by tumor cell after therapy. This analysis is possible due to the decreased hexokinase II enzyme activity in adjacent tissues [86], by reducing ${ }^{18}$ F-FDG uptake. This radiotracer (half-life of $109.7 \mathrm{~min}$ ) is the most widely used for detecting the glucose uptake by tumor cells $[86,87]$. The main objective of using PET analysis in GBM cases is to differentiate tumor recurrence from radiation necrosis [61]. There is a linear relationship in PET signals between GBM cell number and ${ }^{18} \mathrm{~F}-\mathrm{FDG}$ uptake levels by these cells, that is significantly higher in small cell lung cancer with activity [88]. In small animals, ${ }^{18}$ F-FDG reaches tumor internalization plateau approximately after $45 \mathrm{~min}$ of administration $[89,90]$. It is possible that the decrease in glucose consumption evidenced in our study is related to decreased nutritional intake due to antiangiogenic effects. ${ }^{18} \mathrm{~F}$-FDG has been clinically and preclinically tested as a marker to analyze antiangiogenic effects. One study showed the efficacy of ${ }^{18} \mathrm{~F}-\mathrm{FDG}$ analysis correlating the overall survival of 11 gliomas patients with the antiangiogenic effect caused by drugs as bevacizumab [91]. Some preclinical studies are capable 
to detect tumor vascularization [64]. A limitation of our study was the lack of information about antiangiogenic mechanisms since we have not used specific epitopes markers such as HIF1, VEGF, PDGFR $\beta$, among others.

At the same time to the imaging, technique analysis performed evaluations of animals spontaneous locomotion, and this approach is viable, sensitive, and useful for longitudinal evaluations. The motor behavior assessment for the tumor growth analysis and the MHT therapeutic effect was correlated to the BLI and PET results, mainly in the late phase of the MHT evaluation. The F-MOV, S-MOV, F-REA, and S-REA frequencies in tumoral control group showed continuous regression due to the motor impairment related to the tumor evolution, corroborating our group previous study that delineated impairment of motor pattern in GBM tumor model at 7, 14, 21, and 28 days after tumor induction using C6 cells [53]. The animals' behavior promoted by MHT therapeutic was assessed after each MHT application leading to a discrete improvement of movement frequency, but this occurred mainly after the first and second MHT application and was not maintained constantly, only after the third MHT application the animals' behavior improvement was evidenced. The lack of studies with this approach after brain tumor induction associated with MHT therapy did not allowed comparisons of our results in the acute phase and late phase, which have shown motor function improvement only for the group that received three MHT applications.

Therefore, approaching MHT therapeutic processes with multiple applications, using aminosilanecoated SPION allowed an almost total regression of the tumor, and further evaluations after therapy acute phase is necessary to follow the tumor evolution or total regression. BLI, PET, and spontaneous locomotion evaluation techniques were effective in monitoring longitudinally the therapeutic effects of MHT technique.

\section{Materials and Methods}

\subsection{Magnetic Nanoparticles}

The magnetic nanoparticles used in this study were the superparamagnetic iron oxide nanoparticles coated with aminosilane (SPIONa) containing magnetite nucleus $\left(\mathrm{Fe}_{3} \mathrm{O}_{4}\right)$, coupled with functional amine group $\left(\mathrm{NH}_{2}\right)$ (fluidMAG-Amine, Chemicell, Berlin, Germany), with density of $\sim 1.25 \mathrm{~g} / \mathrm{cm}^{3}$, hydrodynamic diameter of $100 \mathrm{~nm}$, zeta potential of $+20 \mathrm{mV}$, and SPION number of $1.8 \times 10^{15} / \mathrm{g}$ approximately.

\subsection{Evaluation of the Heating Potential of SPIONa}

SPIONa $(5 \mathrm{mg} / \mathrm{mL})$ samples dispersed in aqueous medium, allocated in thermally isolated recipient were submitted to MHT process in different combinations of magnetic fields (100, 200 and 300 Gauss) and oscillation frequency $(309,364,420,557$, and $874 \mathrm{kHz}$ ) of the AMF, using the DM100 system (nB nanoScale Biomagnetics, Zaragoza, Spain) until achieving the therapeutic temperature of $43{ }^{\circ} \mathrm{C}$ in order to obtain heating curves as function of time $(\Delta T / \Delta t)$. The samples temperature was monitored by optical fiber (3204, Luxtron Corp., Santa Ana, California, USA). Samples initial temperature was $19^{\circ} \mathrm{C}$, and measurements were performed in quintuplicates.

The specific absorption rate $(S A R)$ values were calculated in accordance with the following equation:

$$
S A R=\frac{m_{\text {SPION }} C_{\text {SPION }}+m_{L} C_{L}}{m_{\text {SPION }}}\left(\frac{\Delta T}{\Delta t}\right)_{\text {max }}
$$

where $m_{S P I O N}$ is the mass of SPION $[\mathrm{kg}] ; C_{S P I O N}$ is the nanoparticle specific heat capacity $[\mathrm{J} /(\mathrm{kg} . \mathrm{K})] ; m_{L}$ is the mass of liquid $[\mathrm{kg}]$, and $C_{L}$ is the medium-specific heat capacity $[\mathrm{J} /(\mathrm{kg} . \mathrm{K})]$. 


\subsection{In Vitro Study}

\subsubsection{C6 Cells Culture}

C6 cells were cultivated in the Dulbecco's Modified Eagle's Medium: Nutrient Mixture F-12 (DMEM/F12) (GIBCO ${ }^{\circledR}$ Invitrogen Corporation, California, USA) supplemented with $12 \%$ fetal bovine serum (FBS) (GIBCO ${ }^{\circledR}$ Invitrogen Corporation, CA, USA) and $1 \%$ antibiotic-antimycotic solution $\left(\mathrm{GIBCO}^{\circledR}\right.$ Invitrogen Corporation, CA, USA). These cells were maintained incubated at $37^{\circ} \mathrm{C}$ and $5 \%$ $\mathrm{CO}_{2}$ in an incubator Forma ${ }^{\mathrm{TM}}$ Series II (Thermo Fisher Scientific, Massachusetts, USA). When necessary these cells were tripsinized using $0.25 \%$ Trypsin-EDTA solution $\left(\mathrm{GIBCO}^{\circledR}\right.$ Invitrogen Corporation, CA, USA). Aiming to evaluate the therapeutic process using the BLI technique, the C6 cells were transduced with luciferase lentiviral vector using an already established protocol [53].

\subsubsection{Kinetics of Bioluminescent Signal of Luciferase Transduced C6 Cells}

For the BLI signal evaluation (the BLI maximum intensity signal and temporal evaluation of BLI signal as a function of cell number), $100 \mu \mathrm{L}$ of D-luciferin $(30 \mathrm{mg} / \mathrm{mL})$ was added in the subsequent C6 cells concentrations: $10^{4} ; 2.5 \times 10^{4} ; 5 \times 10^{4} ; 7.5 \times 10^{4} ; 10^{5} ; 5 \times 10^{5}$, and $10^{6}$ cells/well. BLI signal acquisition was performed using IVIS ${ }^{\circledR}$ Lumina LT Series III equipment (Xenogen Corp, PerkinElmer. California, USA) with the following parameters: expossure time of $2 \mathrm{~ms}$, binning of 2 and $\mathrm{f} / \mathrm{stop}$ of 4 . The BLI images were processed using the Living Image Software version 4.3.1. (IVIS Imaging System) in radiation absolute units (photons/s) through the ROI of $1 \mathrm{~cm}^{2}$.

\subsubsection{Labeling C6 Cells with SPIONa and Internalization Imagining}

Culture wells containing $10^{5} \mathrm{C} 6$ cells were plated with $2 \mathrm{~mL} /$ well of DMEM/F12. After $24 \mathrm{~h}$, in the culture wells was added a concentration of 100 and $200 \mu \mathrm{gFe} / \mathrm{mL}$ of SPIONa (in triplicate) and incubated for $12 \mathrm{~h}$. After incubation, C6 cells were washed with Phosphate Buffered Saline (PBS) $\left(\mathrm{GIBCO}^{\circledR}\right.$ Invitrogen Corporation, California, USA) and fixed for $60 \mathrm{~min}$ with $500 \mu \mathrm{L}$ of paraformaldehyde $4 \%$ (PFA 4\%) (Sigma-Aldrich, Missouri, USA).

The internalization evaluation was performed using prussia blue staining with $500 \mathrm{uL}(5 \%$ potassium ferrocyanide, Sigma-Aldrich, St Louis, MO, USA, and 5\% hydrochloric acid, Merck, Darmstadt, Germany) for $15 \mathrm{~min}$ and washed once with deionized water. Then, the nuclear fast red staining was performed with $1 \%$ (0.02 g of nuclear fast red in $2 \mathrm{~mL}$ of deionized water) for $10 \mathrm{~min}$ for nuclei counterstaining, and then quickly washed once more and analyzed by optical microscopy image. C6 cells stained images were registered using brighfield microscopy Eclipse TI-Nikon (Nikon Corporation, Tokyo, Japan).

\subsubsection{In Vitro MHT}

In vitro MHT process was performed in three stages and therapeutic efficiency was evaluated through the BLI technique as shown in the experimental design displayed in Figure 9A. Samples of $10^{6} \mathrm{C} 6$ cells were labeled with SPIONa for $12 \mathrm{~h}$ followed by washing. These cells were trypsinized, suspended in $40 \mu \mathrm{L}$ of DMEM/F12 with their respective controls groups and submitted to MHT process according to the heating planning (Figure 9B) using the DM100 system that apply AMF to keep constant temperature of $43{ }^{\circ} \mathrm{C}$. According to the SAR values previously calculated were chosen two combinations of magnetic field and oscillation frequencies of AMF [(f1:309 kHz-B:300 Gauss); (f2:557 kHz-B:300 Gauss)] to apply in the MHT process. For MHT process evaluation was used six groups: (i) C6 group, cells unlabeled; (ii) C6-SPION group, cells labeled with SPIONa; (iii) C6-f1-B group, cells unlabeled submitted to AMF(f1-B); (iv) C6-SPION-f1-B group, cells labeled with SPIONa submitted to AMF(f1-B); (v) C6-f2-B group, cells unlabeled submitted to AMF (f2-B); and (vi) C6-SPION-f2-B group, cells labeled with SPIONa submitted to AMF(f2-B); as shown in Figure 9C. The MHT was applied for $30 \mathrm{~min}$ at 0,3 , and 6 days and the temperature monitored using an optical fiber (Luxtron 3204). The BLI evaluations were performed at 2, 5, and 8 days, after the addition of 
$10 \mu \mathrm{l}$ of luciferin/sample and posterior signal quantification in radiation absolute units (photons/s). The experiment was performed in triplicate per experimental group.

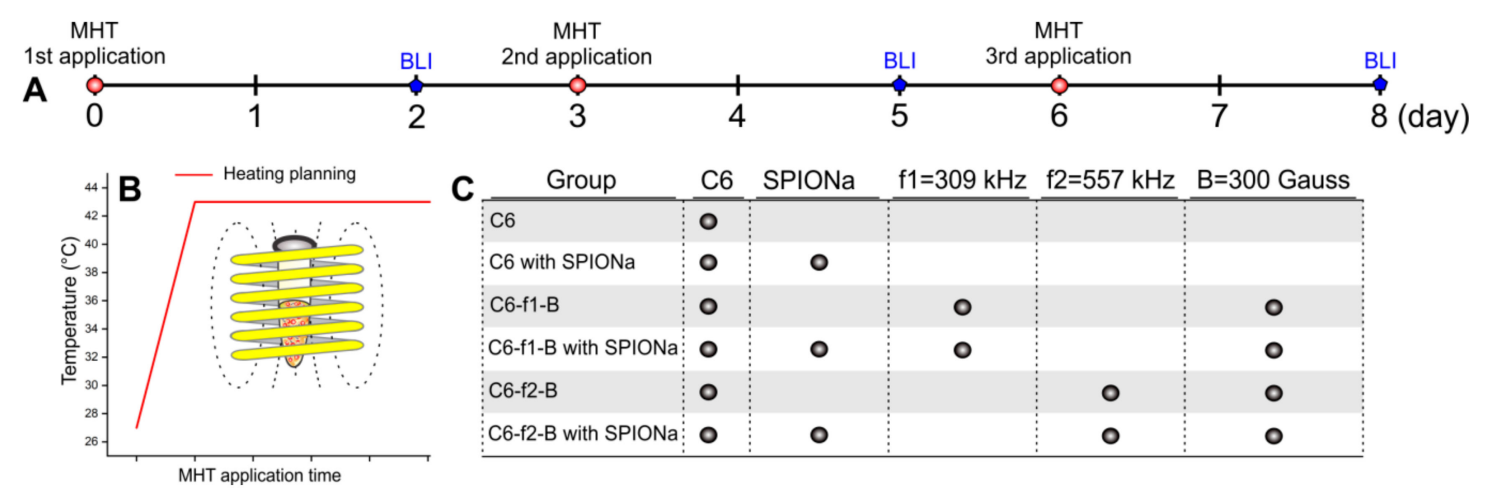

Figure 9. Experimental design of manifold applications of MHT in vitro. (A) Timeline with three MHT applications at 0,3 , and 6 days and their correspondent evaluation using BLI at 2, 5, and 8 days; (B) heating planning of the MHT process, keeping the constant temperature of $43^{\circ} \mathrm{C}$; (C) experimental groups and their respective controls submitted to MHT therapeutic process with two combinations of magnetic field and oscillation frequencies of the AMF [(f1:309 kHz-B:300 Gauss); (f2:557 kHz-B:300 Gauss)].

\subsection{In Vivo Study}

Forty-five male Wistar rats, weighing between 250 to $350 \mathrm{~g}$ were acclimated at the Surgical Experimentation and Training Center (CETEC) of the Instituto de Ensino e Pesquisa Albert Eisntein. These animals were exposed at $21 \pm 2{ }^{\circ} \mathrm{C}$ with a $12 \mathrm{~h} \mathrm{light/dark} \mathrm{cycle.} \mathrm{Access} \mathrm{to} \mathrm{food} \mathrm{and} \mathrm{water} \mathrm{was} \mathrm{ad}$ libitum during the experiment. This vivarium is accredited by the Association for the Assessment and Accreditation of Laboratory Animal Care International (AAALAC International). Our study was approved by the Ethics in Animal Research Committee of the Hospital Israelita Albert Einstein; number 3126-17.

\subsubsection{Glioblastoma Tumor Inducion in Animal Model}

The animals were anesthetized with isoflurane $(2-4 \%)$ by SomnoSuite ${ }^{\circledR}$ system (Kent Scientific Corporation, Torrington, USA) and placed into the Neurostar Robot stereotaxic StereoDrive ${ }^{\circledR}$ instrument (NeuroStar $\mathrm{GmbH}$, Tübingen, Germany), which holds the skull with ear bars and a clamp system that tightens against the frontonasal bone and the palate. After making a skin inscision on the dorsal region of the skull, the system was calibrated using the follow reference points: bregma; lambda and $2 \mathrm{~mm}$ right and left of central point between bregma and lambda. The target injection site was defined by the following stereotaxic coordinates: $2.0 \mathrm{~mm}$ (bregma; rostral-caudal axis), $2.0 \mathrm{~mm}$ (medial-lateral axis), and $3.0 \mathrm{~mm}$ (ventral-dorsal axis) of Paxinos Atlas [92] and $10^{6} \mathrm{C} 6$ cells suspended in $10 \mu \mathrm{l}$ of DMEM/F12 was injected in the 3,6 $\mu \mathrm{L} / \mathrm{min}$, using the Hamilton syringe of $10 \mu \mathrm{L}$ (Hamilton Company, Nevada, USA). The bone recomposition was performed with Dencrilay ${ }^{\circledR}$ (Dencril Produtos Odontológicos, Brazil) diluted in self-curing acrylic based polymethyl methacrylate (Classic Dental Products, Brazil) and posterior suture of the animal.

\subsubsection{Evaluation of Tumor Growth by Histological Analysis}

The histological tumor growth evaluation was performed at $7(n=5), 14(n=5), 21(n=5)$, and 28 days $(n=5)$ after tumor induction, together with the controls without tumor induction $(n=5)$. The brain was extracted and fixed with $4 \%$ of Paraformaldehyde (PFA) for $48 \mathrm{~h}$, then dehydrated in $30 \%$ of sucrose for $48 \mathrm{~h}$ and frozen at $-80^{\circ} \mathrm{C}$. The histological sections were taken with $20 \mu \mathrm{m}$ of thickness at $-20^{\circ} \mathrm{C}$ using the cryostat $\mathrm{CM} 1850^{\circledR}$ (Leica Microsystems, Wetzlar, Germany) and placed in the gelatinized histological blades (Gelatin from porcine skin G2500, Sigma-Aldrich, Missouri, USA) 
for H\&E staining (Sigma-Aldrich, Missouri USA). Blades were digitalized using ScanScope AT Turbo ${ }^{\circledR}$ equipment (Leica Microsystems, Wetzlar, Germany).

\subsubsection{In Vivo Experimental Design of Magnetic Hyperthermia}

For in vivo MHT study experimental design (Figure 10), five experimental groups were established: (i) No_Tumor group, animals submitted to craniectomy and administered $10 \mathrm{uL}$ of saline; (ii) Tumor group, animals submitted to tumor induction without the MHT therapeutic application; (iii) Tumor+1MHT group, animals submitted to tumor induction and one section of MHT therapeutic at 14th day after induction; (iv) Tumor+2MHT group, animals submitted to tumor induction and two sections of the MHT therapeutic at 14th and 17th day after induction; and (v) Tumor+3MHT group, animals submitted to tumor induction and three sections of the MHT therapeutic at the 14th, 17th, and 20th day after induction. The BLI signal evaluation was performed one day before each MHT application, as well as after 2 and 12 days of the third MHT application, having a common period for all groups at 13, 22, and 32 days after tumor induction, as shown in Figure 10. In addition, the MHT therapeutic process was evaluated through glucose metabolism using PET/CT before and after all the MHT process at 13 and 22 days of tumor induction. The behavioral analysis was performed at $0,7,14$, $16,19,24$, and 32 days.

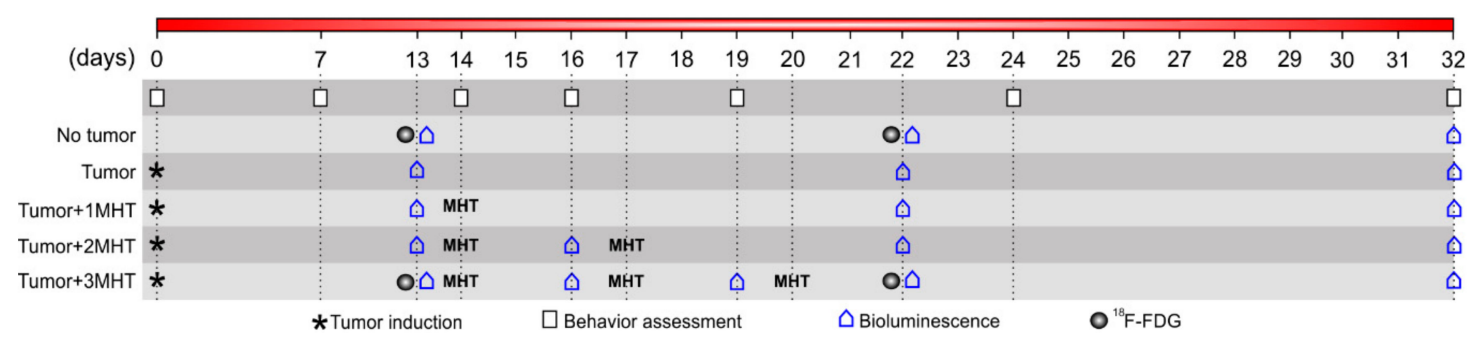

Figure 10. Experimental design, in vivo, for multiple MHT applications performed in 5 experimental groups: No_Tumor group, Tumor group, Tumor+1MHT group, Tumor+2MHT group, and Tumor+3MHT group were evaluated the therapeutic efficiency by BLI, ${ }^{18}$ F-FDG-PET/CT, and behavior.

In Vivo MHT Therapeutic Process

The MHT therapeutic process was evaluated with one, two and three applications at 14,17 , and 20 days after glioblastoma tumor induction (Figure 10). At 13th day of tumor induction was performed the tumor volumetric analysis by BLI, and the next day $40 \mu \mathrm{L}$ of SPIONa was administered in four coordinates around the center of the tumor mass $(1 \mu \mathrm{L} / \mathrm{min})$ using the robotic stereotaxic equipment (NeuroStar $\mathrm{GmbH}$, Tübingen, Germany) $5 \mathrm{~h}$ before the first MHT application. The MHT applications were performed using the best parameters of AMF and oscillation frequency determined by the in vitro study using the DM1000 system. The therapeutic temperature of $43^{\circ} \mathrm{C}$ was monitored by an optical fiber (Luxtron 3204), and kept constant for $30 \mathrm{~min}$.

Therapeutic Process Evaluation by BLI

The MHT therapeutic efficiency was evaluated by BLI signal of tumor mass at 13 days after tumor induction (before MHT application, control measurement) and at 16, 19, and 22 days that correspond to 2 days after each MHT application. In addition, the BLI evaluation was also performed after 12 days of the third MHT application (at 32nd day) in all groups, allowing comparative analysis between groups and their respective controls (Figure 10). For BLI image acquisitions were administered $1500 \mu \mathrm{L}$ of D-luciferin intraperitoneal $(15 \mathrm{mg} / \mathrm{mL})$ and the parameters and analysis of the BLI images were similar to the ones used in the in vitro study. 


\section{Evaluation of MHT Therapeutic Effect by ${ }^{18}$ F-FDG-PET}

The MHT therapeutic effect was also evaluated by glucose metabolism analysis using the PET technique and administration of ${ }^{18} \mathrm{~F}-\mathrm{FDG}$ in the animals. The PET images were performed before (at the 13th day) and after three MHT applications (at the 22nd day), as depicted in Figure 10, using a small animal equipment Triumph ${ }^{\circledR}$ II preclinical scanner (Gamma Medica-Ideas, Nortridge, CA, USA). ${ }^{18} \mathrm{~F}-\mathrm{FDG}$ (38-40 MBq) was injected in the penile vein and after $45 \mathrm{~min}$, the animal was posionited with the brain in the center of the field of view. Image was acquired for $30 \mathrm{~min}$ and reconstructed using OSEM 3D algoritm, 20 interactions, and 4 subgroups. Images were fused to a MRI template available in the PMOD ${ }^{\circledR}$ software.

\section{Evaluation of MHT Therapeutic Effect by Spontaneous Locomotor Activity}

The behavior assessment through the global spontaneous locomotor activity was performed using the Infrared (IR) Actimeter LE 8825 systems (Actitrack, Panlab Harvard Apparatus, Barcelona, Spain), which is basically composed by a 2 dimensional ( $X$ and $Y$ axes) square frame, a frame support and a control unit. Each frame has $16 \times 16$ infrared beams for optimal subject detection. Each animal was posicioned in the center of an arena and during $5 \mathrm{~min}$, they were analysed for spontaneous locomotor activities: frequency of horizontal movement, frequency of vertical moviment and frequency of rearing moviment in slow and fast speed considering $5 \mathrm{~s}$ the time limit of speed classification. The data was acquired at days $0,7,14,16,19,24$, and 32 after tumor induction and processed by SEDACOM v2.0. software (panlab, Barcelona, Spain).

\subsection{Statistic Analysis}

The experimental data were demonstrated by mean and standard deviation and analyzed by JASP (0.9.0.1) and Origin 9.1 software (OriginLab, Northampton, Massachusetts, USA). The data with normal distribution was analyzed with a parametric test and for non normal distribuition was used a non-parametric test. The comparison between two groups was performed using t-student test or Mann-Whitney test and for more than two group comparison or repeated measures was used ANOVA test or Kruskal-Wallis test. In ANOVA test was considered the Post Hoc analysis for more comprehension of the results, comparing the factors two by two. For significative results was considered $p$-value less than 0.05 .

Author Contributions: Conceptualization, G.N.A.R., J.B.M., M.P.N., and L.F.G.; methodology, G.N.A.R., J.B.M., F.A.O., I.S.F., J.M.F., C.C.R., D.d.P.F., P.L.E., D.M.C.F., and L.F.G.; validation, G.N.A.R., M.P.N., J.B.M., F.A.O., I.S.F., and L.F.G.; formal analysis, G.N.A.R., M.P.N., J.M.F., and L.F.G.; investigation, G.N.A.R., M.P.N., J.B.M., J.M.F., D.P., P.L.E., C.C.R., L.E.B.S., and L.F.G.; resources, L.F.G.; data curation, D.T.C., and L.F.G.; writing-original draft preparation, G.N.A.R., M.P.N., J.B.M., F.A.O., L.C.M., C.C.R., C.A.B., and L.F.G.; writing-review and editing, G.N.A.R., M.P.N., J.B.M., F.A.O., L.C.M., and L.F.G.; visualization, G.N.A.R., F.A.O., L.C.M., I.S.F., and L.F.G.; supervision, L.F.G.; project administration, L.F.G.; funding acquisition, L.F.G. All authors have read and agreed to the published version of the manuscript.

Funding: This research was funded by CNPq (400856/2016-6) and FAPESP (2016/21470-3;2014/50983-3).

Conflicts of Interest: The authors declare no conflict of interest.

\section{Abbreviations}

$\begin{array}{ll}\text { MHT } & \text { Magnetic hyperthermia } \\ \text { GBM } & \text { Glioblastoma } \\ \text { SPIONa } & \text { SPION coated with aminosilane } \\ \text { SPION } & \text { Superparamagnetic iron oxide nanoparticle } \\ \text { SAR } & \text { Specific absorption rate } \\ \text { BLI } & \text { Bioluminescence } \\ \text { PET } & \text { Positron emission tomography } \\ \text { TMZ } & \text { Temozolomide } \\ \text { MNP } & \text { Magnetic nanoparticles }\end{array}$




\begin{tabular}{ll} 
AMF & Alternating magnetic field \\
MRI & Magnetic resonance imaging \\
NIRF & Near-infrared fluorescence \\
SPECT & Single Photon Emission Computed Tomography \\
MPI & Magnetic particle imaging \\
CT & Computed tomography \\
ATP & Adenosinetriphosphate \\
2D & Two-dimensional \\
18F-FDG & 18F-2-fluoro-2-deoxy-D-glucose \\
SD & Standard deviation \\
S-MOV & Slow horizontal movement \\
F-MOV & Fast horizontal movement \\
S-REA & Slow rearing \\
F-REA & Fast rearing \\
DH & Hydrodynamic diameter \\
ILP & Intrinsic loss power \\
PEI & Polyethyleneimine \\
Hsp & Heat shock protein \\
MCL & Magnetite cationic liposomes \\
DMEM/F12 & Dulbecco's modified eagle's medium: nutrient mixture F-12 \\
FBS & Fetal bovine serum \\
PBS & Phosphate buffered saline \\
PFA & Paraformaldehyde \\
IR & Infrared \\
\hline
\end{tabular}

\section{Appendix A}

Table A1. Post Hoc analysis of SAR values obtained when submitted to the magnetic field of 100, 200 and 300 Gauss and frequency oscillation of 309, 364, 420, 557 and $874 \mathrm{kHz}$.

\begin{tabular}{|c|c|c|c|c|}
\hline \multicolumn{2}{|c|}{$\begin{array}{l}\text { SAR Value Obtained for Frequency and } \\
\text { Magnetic Field Combinations }\end{array}$} & \multirow{2}{*}{$\begin{array}{c}\text { Mean Difference } \\
-50.968\end{array}$} & \multirow{2}{*}{$\begin{array}{c}\text { Standard Error } \\
4.637\end{array}$} & \multirow{2}{*}{$\begin{array}{c}p \text { Bonferroni } \\
<0.001\end{array}$} \\
\hline $309 \mathrm{kHz}-100 \mathrm{G}$ & $309 \mathrm{kHz}-200 \mathrm{G}$ & & & \\
\hline & $309 \mathrm{kHz}-300 \mathrm{G}$ & -165.508 & 4.637 & $<0.001$ \\
\hline & $364 \mathrm{kHz}-100 \mathrm{G}$ & -2.997 & 4.637 & 1.000 \\
\hline & $364 \mathrm{kHz}-200 \mathrm{G}$ & -58.007 & 4.637 & $<0.001$ \\
\hline & $364 \mathrm{kHz}-300 \mathrm{G}$ & -181.675 & 4.637 & $<0.001$ \\
\hline & $420 \mathrm{kHz}-100 \mathrm{G}$ & -4.506 & 4.637 & 1.000 \\
\hline & $420 \mathrm{kHz}-200 \mathrm{G}$ & -75.329 & 4.637 & $<0.001$ \\
\hline & $420 \mathrm{kHz}-300 \mathrm{G}$ & -234.986 & 4.637 & $<0.001$ \\
\hline & $557 \mathrm{kHz}-100 \mathrm{G}$ & -7.290 & 4.637 & 1.000 \\
\hline & $557 \mathrm{kHz}-200 \mathrm{G}$ & -78.983 & 4.637 & $<0.001$ \\
\hline & $557 \mathrm{kHz}-300 \mathrm{G}$ & -316.281 & 4.637 & $<0.001$ \\
\hline & 874 kHz-100 G & -316.281 & 4.637 & 0.542 \\
\hline & $874 \mathrm{kHz}-200 \mathrm{G}$ & -316.281 & 4.637 & $<0.001$ \\
\hline \multirow[t]{4}{*}{309 kHz-200 G } & $309 \mathrm{kHz}-300 \mathrm{G}$ & -114.540 & 4.637 & $<0.001$ \\
\hline & $364 \mathrm{kHz}-100 \mathrm{G}$ & 47.972 & 4.637 & $<0.001$ \\
\hline & $364 \mathrm{kHz}-200 \mathrm{G}$ & -7.039 & 4.637 & 1.000 \\
\hline & $364 \mathrm{kHz}-300 \mathrm{G}$ & -130.707 & 4.637 & $<0.001$ \\
\hline
\end{tabular}


Table A1. Cont.

\begin{tabular}{|c|c|c|c|}
\hline $\begin{array}{l}\text { SAR Value Obtained for Frequency and } \\
\text { Magnetic Field Combinations }\end{array}$ & Mean Difference & Standard Error & $p$ Bonferroni \\
\hline $420 \mathrm{kHz}-100 \mathrm{G}$ & 46.462 & 4.637 & $<0.001$ \\
\hline $420 \mathrm{kHz}-200 \mathrm{G}$ & -24.360 & 4.637 & $<0.001$ \\
\hline $420 \mathrm{kHz}-300 \mathrm{G}$ & -184.018 & 4.637 & $<0.001$ \\
\hline $557 \mathrm{kHz}-100 \mathrm{G}$ & 43.679 & 4.637 & $<0.001$ \\
\hline $557 \mathrm{kHz}-200 \mathrm{G}$ & -28.015 & 4.637 & $<0.001$ \\
\hline $557 \mathrm{kHz}-300 \mathrm{G}$ & -265.313 & 4.637 & $<0.001$ \\
\hline $874 \mathrm{kHz}-100 \mathrm{G}$ & -265.313 & 4.637 & $<0.001$ \\
\hline $874 \mathrm{kHz}-200 \mathrm{G}$ & -265.313 & 4.637 & $<0.001$ \\
\hline \multirow[t]{11}{*}{$309 \mathrm{kHz}-300 \mathrm{G}$} & 162.511 & 4.637 & $<0.001$ \\
\hline & 107.501 & 4.637 & $<0.001$ \\
\hline & -16.167 & 4.637 & 0.087 \\
\hline & 161.001 & 4.637 & $<0.001$ \\
\hline & 90.179 & 4.637 & $<0.001$ \\
\hline & -69.478 & 4.637 & $<0.001$ \\
\hline & 158.218 & 4.637 & $<0.001$ \\
\hline & 86.525 & 4.637 & $<0.001$ \\
\hline & -150.773 & 4.637 & $<0.001$ \\
\hline & -150.773 & 4.637 & $<0.001$ \\
\hline & -150.773 & 4.637 & 1.000 \\
\hline \multirow[t]{9}{*}{$364 \mathrm{kHz}-100 \mathrm{G}$} & -55.011 & 4.637 & $<0.001$ \\
\hline & -178.678 & 4.637 & $<0.001$ \\
\hline & -1.510 & 4.637 & 1.000 \\
\hline & -72.332 & 4.637 & $<0.001$ \\
\hline & -231.990 & 4.637 & $<0.001$ \\
\hline & -4.293 & 4.637 & 1.000 \\
\hline & -75.986 & 4.637 & $<0.001$ \\
\hline & -313.284 & 4.637 & $<0.001$ \\
\hline & -313.284 & 4.637 & 1.000 \\
\hline $874 \mathrm{kHz}-200 \mathrm{G}$ & -313.284 & 4.637 & $<0.001$ \\
\hline \multirow[t]{8}{*}{$364 \mathrm{kHz}-200 \mathrm{G}$} & -123.668 & 4.637 & $<0.001$ \\
\hline & 53.501 & 4.637 & $<0.001$ \\
\hline & -17.321 & 4.637 & 0.040 \\
\hline & -176.979 & 4.637 & $<0.001$ \\
\hline & 50.718 & 4.637 & $<0.001$ \\
\hline & -20.976 & 4.637 & 0.003 \\
\hline & -258.274 & 4.637 & $<0.001$ \\
\hline & -258.274 & 4.637 & $<0.001$ \\
\hline $874 \mathrm{kHz}-200 \mathrm{G}$ & -258.274 & 4.637 & $<0.001$ \\
\hline
\end{tabular}


Table A1. Cont.

\begin{tabular}{|c|c|c|c|c|}
\hline \multicolumn{2}{|c|}{$\begin{array}{l}\text { SAR Value Obtained for Frequency and } \\
\text { Magnetic Field Combinations }\end{array}$} & \multirow{2}{*}{$\begin{array}{c}\text { Mean Difference } \\
177.169 \\
\end{array}$} & \multirow{2}{*}{$\begin{array}{c}\text { Standard Error } \\
4.637 \\
\end{array}$} & \multirow{2}{*}{$\begin{array}{c}p \text { Bonferroni } \\
<0.001 \\
\end{array}$} \\
\hline $364 \mathrm{kHz}-300 \mathrm{G}$ & $420 \mathrm{kHz}-100 \mathrm{G}$ & & & \\
\hline & $420 \mathrm{kHz}-200 \mathrm{G}$ & 106.346 & 4.637 & $<0.001$ \\
\hline & $420 \mathrm{kHz}-300 \mathrm{G}$ & -53.311 & 4.637 & $<0.001$ \\
\hline & 557 kHz-100 G & 174.385 & 4.637 & $<0.001$ \\
\hline & $557 \mathrm{kHz}-200 \mathrm{G}$ & 102.692 & 4.637 & $<0.001$ \\
\hline & $557 \mathrm{kHz}-300 \mathrm{G}$ & -134.606 & 4.637 & $<0.001$ \\
\hline & 874 kHz-100 G & -134.606 & 4.637 & $<0.001$ \\
\hline & $874 \mathrm{kHz}-200 \mathrm{G}$ & -134.606 & 4.637 & 1.000 \\
\hline \multirow[t]{7}{*}{$420 \mathrm{kHz}-100 \mathrm{G}$} & $420 \mathrm{kHz}-200 \mathrm{G}$ & -70.822 & 4.637 & $<0.001$ \\
\hline & $420 \mathrm{kHz}-300 \mathrm{G}$ & -230.480 & 4.637 & $<0.001$ \\
\hline & $557 \mathrm{kHz}-100 \mathrm{G}$ & -2.783 & 4.637 & 1.000 \\
\hline & $557 \mathrm{kHz}-200 \mathrm{G}$ & -74.477 & 4.637 & $<0.001$ \\
\hline & $557 \mathrm{kHz}-300 \mathrm{G}$ & -311.775 & 4.637 & $<0.001$ \\
\hline & 874 kHz-100 G & -311.775 & 4.637 & 1.000 \\
\hline & 874 kHz-200 G & -311.775 & 4.637 & $<0.001$ \\
\hline \multirow[t]{6}{*}{$420 \mathrm{kHz}-200 \mathrm{G}$} & $420 \mathrm{kHz}-300 \mathrm{G}$ & -159.658 & 4.637 & $<0.001$ \\
\hline & $557 \mathrm{kHz}-100 \mathrm{G}$ & 68.039 & 4.637 & $<0.001$ \\
\hline & $557 \mathrm{kHz}-200 \mathrm{G}$ & -3.654 & 4.637 & 1.000 \\
\hline & $557 \mathrm{kHz}-300 \mathrm{G}$ & -240.952 & 4.637 & $<0.001$ \\
\hline & $874 \mathrm{kHz}-100 \mathrm{G}$ & -240.952 & 4.637 & $<0.001$ \\
\hline & $874 \mathrm{kHz}-200 \mathrm{G}$ & -240.952 & 4.637 & $<0.001$ \\
\hline \multirow[t]{5}{*}{$420 \mathrm{kHz}-300 \mathrm{G}$} & $557 \mathrm{kHz}-100 \mathrm{G}$ & 227.697 & 4.637 & $<0.001$ \\
\hline & $557 \mathrm{kHz}-200 \mathrm{G}$ & 156.003 & 4.637 & $<0.001$ \\
\hline & $557 \mathrm{kHz}-300 \mathrm{G}$ & -81.295 & 4.637 & $<0.001$ \\
\hline & $874 \mathrm{kHz}-100 \mathrm{G}$ & -81.295 & 4.637 & $<0.001$ \\
\hline & $874 \mathrm{kHz}-200 \mathrm{G}$ & -81.295 & 4.637 & $<0.001$ \\
\hline \multirow[t]{4}{*}{$557 \mathrm{kHz}-100 \mathrm{G}$} & $557 \mathrm{kHz}-200 \mathrm{G}$ & -71.693 & 4.637 & $<0.001$ \\
\hline & $557 \mathrm{kHz}-300 \mathrm{G}$ & -308.991 & 4.637 & $<0.001$ \\
\hline & $874 \mathrm{kHz}-100 \mathrm{G}$ & -308.991 & 4.637 & 1.000 \\
\hline & $874 \mathrm{kHz}-200 \mathrm{G}$ & -308.991 & 4.637 & $<0.001$ \\
\hline \multirow[t]{3}{*}{$557 \mathrm{kHz}-200 \mathrm{G}$} & $557 \mathrm{kHz}-300 \mathrm{G}$ & -237.298 & 4.637 & $<0.001$ \\
\hline & $874 \mathrm{kHz}-100 \mathrm{G}$ & -237.298 & 4.637 & $<0.001$ \\
\hline & $874 \mathrm{kHz}-200 \mathrm{G}$ & -237.298 & 4.637 & $<0.001$ \\
\hline \multirow[t]{2}{*}{$557 \mathrm{kHz}-300 \mathrm{G}$} & $874 \mathrm{kHz}-100 \mathrm{G}$ & -237.298 & 4.637 & $<0.001$ \\
\hline & $874 \mathrm{kHz}-200 \mathrm{G}$ & -237.298 & 4.637 & $<0.001$ \\
\hline 874 kHz-100 G & $874 \mathrm{kHz}-200 \mathrm{G}$ & -237.298 & 4.637 & $<0.001$ \\
\hline
\end{tabular}


Table A2. Post Hoc analysis of the BLI signal of experimental groups in the first MHT application.

\begin{tabular}{|c|c|c|c|c|c|}
\hline \multicolumn{2}{|c|}{ Groups } & \multirow{3}{*}{$\begin{array}{c}\begin{array}{c}\text { Mean } \\
\text { Difference }\end{array} \\
4.000 \times 10^{7}\end{array}$} & \multicolumn{2}{|c|}{ 95\% IC of Mean Difference } & \multirow{3}{*}{$\begin{array}{c}p \\
1.000\end{array}$} \\
\hline & & & \multirow{2}{*}{$\begin{array}{c}\text { Inferior } \\
-4.279 \times 10^{8}\end{array}$} & \multirow{2}{*}{$\begin{array}{c}\text { Superior } \\
5.079 \times 10^{8} \\
\end{array}$} & \\
\hline C6 without SPIONa & C6 with SPIONa & & & & \\
\hline & C6-f1-B without SPIONa & $-2.950 \times 10^{8}$ & $-7.629 \times 10^{8}$ & $1.729 \times 10^{8}$ & 0.906 \\
\hline & C6-f1-B with SPIONa & $2.323 \times 10^{9}$ & $1.855 \times 10^{9}$ & $2.790 \times 10^{9}$ & $<0.001$ \\
\hline & C6-f2-B without SPIONa & $-3.050 \times 10^{8}$ & $-7.729 \times 10^{8}$ & $1.629 \times 10^{8}$ & 0.794 \\
\hline & C6-f2-B with SPIONa & $3.197 \times 10^{9}$ & $2.729 \times 10^{9}$ & $3.665 \times 10^{9}$ & $<0.001$ \\
\hline \multirow[t]{4}{*}{ C6 with SPIONa } & C6-f1-B without SPIONa & $-3.350 \times 10^{8}$ & $-8.029 \times 10^{8}$ & $1.329 \times 10^{8}$ & 0.530 \\
\hline & C6-f1-B with SPIONa & $2.283 \times 10^{9}$ & $1.815 \times 10^{9}$ & $2.750 \times 10^{9}$ & $<0.001$ \\
\hline & C6-f2-B without SPIONa & $-3.450 \times 10^{8}$ & $-8.129 \times 10^{8}$ & $1.229 \times 10^{8}$ & 0.462 \\
\hline & C6-f2-B with SPIONa & $3.157 \times 10^{9}$ & $2.689 \times 10^{9}$ & $3.625 \times 10^{9}$ & $<0.001$ \\
\hline \multirow[t]{3}{*}{$\begin{array}{l}\text { C6-f1-B without } \\
\text { SPIONa }\end{array}$} & C6-f1-B with SPIONa & $2.618 \times 10^{9}$ & $2.150 \times 10^{9}$ & $3.085 \times 10^{9}$ & $<0.001$ \\
\hline & C6-f2-B without SPIONa & $-1.000 \times 10^{7}$ & $-4.779 \times 10^{8}$ & $4.579 \times 10^{8}$ & 1.000 \\
\hline & C6-f2-B with SPIONa & $3.492 \times 10^{9}$ & $3.024 \times 10^{9}$ & $3.960 \times 10^{9}$ & $<0.001$ \\
\hline \multirow[t]{2}{*}{ C6-f1-B with SPIONa } & C6-f2-B without SPIONa & $-2.628 \times 10^{9}$ & $-3.095 \times 10^{9}$ & $-2.160 \times 10^{9}$ & $<0.001$ \\
\hline & C6-f2-B with SPIONa & $8.745 \times 10^{8}$ & $4.066 \times 10^{8}$ & $1.342 \times 10^{9}$ & $<0.001$ \\
\hline $\begin{array}{l}\text { C6-f2-B without } \\
\text { SPIONa }\end{array}$ & C6-f2-B with SPIONa & $3.502 \times 10^{9}$ & $3.034 \times 10^{9}$ & $3.970 \times 10^{9}$ & $<0.001$ \\
\hline
\end{tabular}

Table A3. Post Hoc analysis of the BLI signal of experimental groups in the second MHT application.

\begin{tabular}{|c|c|c|c|c|c|}
\hline \multicolumn{2}{|c|}{ Groups } & \multirow{3}{*}{$\begin{array}{c}\begin{array}{c}\text { Mean } \\
\text { Difference }\end{array} \\
5.388 \times 10^{6} \\
\end{array}$} & \multicolumn{2}{|c|}{ 95\% IC of Mean Difference } & \multirow{3}{*}{$\begin{array}{c}p \\
1.000\end{array}$} \\
\hline & & & \multirow{2}{*}{$\begin{array}{c}\text { Inferior } \\
-6.056 \times 10^{8}\end{array}$} & \multirow{2}{*}{$\begin{array}{c}\text { Superior } \\
6.164 \times 10^{8} \\
\end{array}$} & \\
\hline C6 without SPIONa & C6 with SPIONa & & & & \\
\hline & C6-f1-B without SPIONa & $5.383 \times 10^{6}$ & $-6.056 \times 10^{8}$ & $6.164 \times 10^{8}$ & 1.000 \\
\hline & C6-f1-B with SPIONa & $3.933 \times 10^{9}$ & $3.322 \times 10^{9}$ & $4.544 \times 10^{9}$ & $<0.001$ \\
\hline & C6-f2-B without SPIONa & $5.388 \times 10^{6}$ & $-6.056 \times 10^{8}$ & $6.164 \times 10^{8}$ & 1.000 \\
\hline & C6-f2-B with SPIONa & $4.588 \times 10^{9}$ & $3.977 \times 10^{9}$ & $5.199 \times 10^{9}$ & $<0.001$ \\
\hline \multirow[t]{4}{*}{ C6 with SPIONa } & C6-f1-B without SPIONa & -4656.000 & $-6.110 \times 10^{8}$ & $6.110 \times 10^{8}$ & 1.000 \\
\hline & C6-f1-B with SPIONa & $3.928 \times 10^{9}$ & $3.317 \times 10^{9}$ & $4.539 \times 10^{9}$ & $<0.001$ \\
\hline & C6-f2-B without SPIONa & 0.000 & $-6.110 \times 10^{8}$ & $6.110 \times 10^{8}$ & 1.000 \\
\hline & C6-f2-B with SPIONa & $4.582 \times 10^{9}$ & $3.971 \times 10^{9}$ & $5.193 \times 10^{9}$ & $<0.001$ \\
\hline \multirow[t]{3}{*}{$\begin{array}{l}\text { C6-f1-B without } \\
\text { SPIONa }\end{array}$} & C6-f1-B with SPIONa & $3.928 \times 10^{9}$ & $3.317 \times 10^{9}$ & $4.539 \times 10^{9}$ & $<0.001$ \\
\hline & C6-f2-B without SPIONa & 4656.000 & $-6.110 \times 10^{8}$ & $6.110 \times 10^{8}$ & 1.000 \\
\hline & C6-f2-B with SPIONa & $4.582 \times 10^{9}$ & $3.971 \times 10^{9}$ & $5.193 \times 10^{9}$ & $<0.001$ \\
\hline \multirow[t]{2}{*}{ C6-f1-B with SPIONa } & C6-f2-B without SPIONa & $-3.928 \times 10^{9}$ & $-4.539 \times 10^{9}$ & $\begin{array}{c}-3.317 \times \\
109\end{array}$ & $<0.001$ \\
\hline & C6-f2-B with SPIONa & $6.546 \times 108$ & $4.360 \times 107$ & $1.266 \times 109$ & 0.047 \\
\hline $\begin{array}{l}\text { C6-f2-B without } \\
\text { SPIONa }\end{array}$ & C6-f2-B with SPIONa & $4.582 \times 109$ & $3.971 \times 109$ & $5.193 \times 109$ & $<0.001$ \\
\hline
\end{tabular}


Table A4. Post Hoc analysis of the BLI signal of experimental groups in the third MHT application.

\begin{tabular}{cccccc}
\hline & \multirow{2}{*}{ Groups } & Mean & \multicolumn{2}{c}{$\mathbf{9 5 \% \text { IC of Mean Difference }}$} & $p$ \\
\cline { 5 - 6 } & & Difference & Inferior & Superior & \\
\hline C6 without SPIONa & C6 with SPIONa & $-8.728 \times 10^{7}$ & $-7.977 \times 10^{8}$ & $6.231 \times 10^{8}$ & 1.000 \\
\hline & C6-f1-B without SPIONa & $-2.182 \times 10^{8}$ & $-9.286 \times 10^{8}$ & $4.922 \times 10^{8}$ & 1.000 \\
\hline & C6-f1-B with SPIONa & $5.979 \times 10^{9}$ & $5.268 \times 10^{9}$ & $6.689 \times 10^{9}$ & $<0.001$ \\
\hline & C6-f2-B without SPIONa & $-1.746 \times 10^{8}$ & $-8.850 \times 10^{8}$ & $5.358 \times 10^{8}$ & 1.000 \\
\hline C6 with SPIONa & C6-f2-B with SPIONa & $5.455 \times 10^{9}$ & $4.745 \times 10^{9}$ & $6.165 \times 10^{9}$ & $<0.001$ \\
\hline & C6-f1-B without SPIONa & $-1.309 \times 10^{8}$ & $-8.413 \times 10^{8}$ & $5.795 \times 10^{8}$ & 1.000 \\
\hline C6-f1-B with SPIONa & $6.066 \times 10^{9}$ & $5.356 \times 10^{9}$ & $6.776 \times 10^{9}$ & $<0.001$ \\
\hline C6-f1-B without & C6-f2-B with SPIONa & $5.542 \times 10^{9}$ & $4.832 \times 10^{9}$ & $6.253 \times 10^{9}$ & $<0.001$ \\
\hline SPIONa & C6-f1-B with SPIONa & $6.197 \times 10^{9}$ & $5.486 \times 10^{9}$ & $6.907 \times 10^{9}$ & $<0.001$ \\
\hline & C6-f2-B without SPIONa & $4.363 \times 10^{7}$ & $-6.668 \times 10^{8}$ & $7.540 \times 10^{8}$ & 1.000 \\
\hline C6-f1-B with SPIONa & C6-f2-B without SPIONa & $-6.153 \times 10^{9}$ & $-6.864 \times 10^{9}$ & $-5.443 \times 10^{9}$ & $<0.001$ \\
\hline & C6-f2-B with SPIONa & $-5.237 \times 10^{8}$ & $-1.234 \times 10^{9}$ & $1.867 \times 10^{8}$ & 0.463 \\
\hline $\begin{array}{c}\text { C6-f2-B without } \\
\text { SPIONa }\end{array}$ & C6-f2-B with SPIONa & $5.630 \times 10^{9}$ & $4.919 \times 10^{9}$ & $6.340 \times 10^{9}$ & $<0.001$ \\
\hline
\end{tabular}

\section{References}

1. Louis, D.N.; Perry, A.; Reifenberger, G.; von Deimling, A.; Figarella-Branger, D.; Cavenee, W.K.; Ohgaki, H.; Wiestler, O.D.; Kleihues, P.; Ellison, D.W. The 2016 World Health Organization Classification of Tumors of the Central Nervous System: A summary. Acta Neuropathol. 2016, 131, 803-820. [CrossRef] [PubMed]

2. Pisapia, D.J. The Updated World Health Organization Glioma Classification: Cellular and Molecular Origins of Adult Infiltrating Gliomas. Arch. Pathol. Lab. Med. 2017, 141, 1633-1645. [CrossRef] [PubMed]

3. Ostrom, Q.T.; Gittleman, H.; Truitt, G.; Boscia, A.; Kruchko, C.; Barnholtz-Sloan, J.S. CBTRUS Statistical Report: Primary Brain and Other Central Nervous System Tumors Diagnosed in the United States in 2011-2015. Neuro-Oncol. 2018, 20, iv1-iv86. [CrossRef] [PubMed]

4. Stupp, R.; Mason, W.P.; van den Bent, M.J.; Weller, M.; Fisher, B.; Taphoorn, M.J.; Belanger, K.; Brandes, A.A.; Marosi, C.; Bogdahn, U.; et al. Radiotherapy plus concomitant and adjuvant temozolomide for glioblastoma. N. Engl. J. Med. 2005, 352, 987-996. [CrossRef] [PubMed]

5. Braun, K.; Ahluwalia, M.S. Treatment of Glioblastoma in Older Adults. Curr. Oncol. Rep. 2017, 19, 81. [CrossRef]

6. Spirou, S.V.; Basini, M.; Lascialfari, A.; Sangregorio, C.; Innocenti, C. Magnetic Hyperthermia and Radiation Therapy: Radiobiological Principles and Current Practice (dagger). Nanomaterials 2018, 8, 401. [CrossRef]

7. Dadfar, S.M.; Roemhild, K.; Drude, N.I.; von Stillfried, S.; Knüchel, R.; Kiessling, F.; Lammers, T. Iron oxide nanoparticles: Diagnostic, therapeutic and theranostic applications. Adv. Drug Deliv. Rev. 2019, 138, 302-325. [CrossRef]

8. Kuchma, E.; Kubrin, S.; Soldatov, A. The Local Atomic Structure of Colloidal Superparamagnetic Iron Oxide Nanoparticles for Theranostics in Oncology. Biomedicines 2018, 6, 78. [CrossRef]

9. Zhu, X.M.; Wang, Y.X.; Leung, K.C.; Lee, S.F.; Zhao, F.; Wang, D.W.; Lai, J.M.; Wan, C.; Cheng, C.H.; Ahuja, A.T. Enhanced cellular uptake of aminosilane-coated superparamagnetic iron oxide nanoparticles in mammalian cell lines. Int. J. Nanomed. 2012, 7, 953-964. [CrossRef]

10. Noha, S.-H.; Moona, S.H.; Shina, T.-H.; Lima, Y.; Cheona, J. Recent advances of magneto-thermal capabilities of nanoparticles: From design principles to biomedical applications. Nano Today 2017, 13, 61-76. [CrossRef]

11. Feng, Q.; Liu, Y.; Huang, J.; Chen, K.; Huang, J.; Xiao, K. Uptake, distribution, clearance, and toxicity of iron oxide nanoparticles with different sizes and coatings. Sci. Rep. 2018, 8, 2082. [CrossRef] [PubMed]

12. Sun, Z.; Yathindranath, V.; Worden, M.; Thliveris, J.A.; Chu, S.; Parkinson, F.E.; Hegmann, T.; Miller, D.W. Characterization of cellular uptake and toxicity of aminosilane-coated iron oxide nanoparticles with different 
charges in central nervous system-relevant cell culture models. Int. J. Nanomed. 2013, 8, 961-970. [CrossRef] [PubMed]

13. Ito, A.; Shinkai, M.; Honda, H.; Yoshikawa, K.; Saga, S.; Wakabayashi, T.; Yoshida, J.; Kobayashi, T. Heat shock protein 70 expression induces antitumor immunity during intracellular hyperthermia using magnetite nanoparticles. Cancer Immunol. Immunother. 2003, 52, 80-88. [CrossRef] [PubMed]

14. Yanase, M.; Shinkai, M.; Honda, H.; Wakabayashi, T.; Yoshida, J.; Kobayashi, T. Intracellular hyperthermia for cancer using magnetite cationic liposomes: Ex vivo study. Jpn. J. Cancer Res. 1997, 88, 630-632. [CrossRef]

15. Yanase, M.; Shinkai, M.; Honda, H.; Wakabayashi, T.; Yoshida, J.; Kobayashi, T. Antitumor immunity induction by intracellular hyperthermia using magnetite cationic liposomes. Jpn. J. Cancer Res. 1998, 89, 775-782. [CrossRef]

16. Rabias, I.; Tsitrouli, D.; Karakosta, E.; Kehagias, T.; Diamantopoulos, G.; Fardis, M.; Stamopoulos, D.; Maris, T.G.; Falaras, P.; Zouridakis, N.; et al. Rapid magnetic heating treatment by highly charged maghemite nanoparticles on Wistar rats exocranial glioma tumors at microliter volume. Biomicrofluidics 2010, 4. [CrossRef]

17. Ito, A.; Shinkai, M.; Honda, H.; Kobayashi, T. Heat-inducible TNF-alpha gene therapy combined with hyperthermia using magnetic nanoparticles as a novel tumor-targeted therapy. Cancer Gene Ther. 2001, 8, 649-654. [CrossRef]

18. Van Landeghem, F.K.; Maier-Hauff, K.; Jordan, A.; Hoffmann, K.T.; Gneveckow, U.; Scholz, R.; Thiesen, B.; Bruck, W.; von Deimling, A. Post-mortem studies in glioblastoma patients treated with thermotherapy using magnetic nanoparticles. Biomaterials 2009, 30, 52-57. [CrossRef]

19. Gupta, R.; Sharma, D. Biofunctionalization of magnetite nanoparticles with stevioside: Effect on the size and thermal behaviour for use in hyperthermia applications. Int. J. Hyperth. 2019. [CrossRef]

20. Maier-Hauff, K.; Rothe, R.; Scholz, R.; Gneveckow, U.; Wust, P.; Thiesen, B.; Feussner, A.; von Deimling, A.; Waldoefner, N.; Felix, R.; et al. Intracranial thermotherapy using magnetic nanoparticles combined with external beam radiotherapy: Results of a feasibility study on patients with glioblastoma multiforme. J. Neurooncol. 2007, 81, 53-60. [CrossRef]

21. Maier-Hauff, K.; Ulrich, F.; Nestler, D.; Niehoff, H.; Wust, P.; Thiesen, B.; Orawa, H.; Budach, V.; Jordan, A. Efficacy and safety of intratumoral thermotherapy using magnetic iron-oxide nanoparticles combined with external beam radiotherapy on patients with recurrent glioblastoma multiforme. J. Neurooncol. 2011, 103, 317-324. [CrossRef]

22. Alphandéry, E.; Idbaih, A.; Adam, C.; Delattre, J.Y.; Schmitt, C.; Guyot, F.; Chebbi, I. Development of non-pyrogenic magnetosome minerals coated with poly-l-lysine leading to full disappearance of intracranial U87-Luc glioblastoma in 100\% of treated mice using magnetic hyperthermia. Biomaterials 2017, 141, 210-222. [CrossRef] [PubMed]

23. Tsiapa, I.; Efthimiadou, E.K.; Fragogeorgi, E.; Loudos, G.; Varvarigou, A.D.; Bouziotis, P.; Kordas, G.C.; Mihailidis, D.; Nikiforidis, G.C.; Xanthopoulos, S.; et al. 99mTc-labeled aminosilane-coated iron oxide nanoparticles for molecular imaging of $\alpha v \beta 3$-mediated tumor expression and feasibility for hyperthermia treatment. J. Colloid Interface Sci. 2014, 433, 163-175. [CrossRef] [PubMed]

24. Alphandéry, E.; Idbaih, A.; Adam, C.; Delattre, J.Y.; Schmitt, C.; Guyot, F.; Chebbi, I. Chains of magnetosomes with controlled endotoxin release and partial tumor occupation induce full destruction of intracranial U87-Luc glioma in mice under the application of an alternating magnetic field. J. Control. Release 2017, 262, 259-272. [CrossRef] [PubMed]

25. Huang, W.C.; Lu, I.L.; Chiang, W.H.; Lin, Y.W.; Tsai, Y.C.; Chen, H.H.; Chang, C.W.; Chiang, C.S.; Chiu, H.C. Tumortropic adipose-derived stem cells carrying smart nanotherapeutics for targeted delivery and dual-modality therapy of orthotopic glioblastoma. J. Control. Release 2017, 254, 119-130. [CrossRef] [PubMed]

26. Cheng, Y.; Muroski, M.E.; Petit, D.C.M.C.; Mansell, R.; Vemulkar, T.; Morshed, R.A.; Han, Y.; Balyasnikova, I.V.; Horbinski, C.M.; Huang, X.; et al. Rotating magnetic field induced oscillation of magnetic particles for in vivo mechanical destruction of malignant glioma. J. Control. Release 2016, 223, 75-84. [CrossRef]

27. Coisson, M.; Barrera, G.; Celegato, F.; Martino, L.; Kane, S.N.; Raghuvanshi, S.; Vinai, F.; Tiberto, P. Hysteresis losses and specific absorption rate measurements in magnetic nanoparticles for hyperthermia applications. Biochim. Biophys. Acta Gen. Subj. 2017, 1861, 1545-1558. [CrossRef]

28. Zamora-Mora, V.; Fernández-Gutiérrez, M.; González-Gómez, Á.; Sanz, B.; Román, J.S.; Goya, G.F.; Hernández, R.; Mijangos, C. Chitosan nanoparticles for combined drug delivery and magnetic hyperthermia: From preparation to in vitro studies. Carbohydr. Polym. 2017, 157, 361-370. [CrossRef] 
29. Pala, K.; Serwotka, A.; Jeleń, F.; Jakimowicz, P.; Otlewski, J. Tumor-specifc hyperthermia with aptamer-tagged superparamagnetic nanoparticles. Int. J. Nanomed. 2013, 9, 67-76. [CrossRef]

30. Tapeinos, C.; Marino, A.; Battaglini, M.; Migliorin, S.; Brescia, R.; Scarpellini, A.; De Julián Fernández, C.; Prato, M.; Drago, F.; Ciofani, G. Stimuli-responsive lipid-based magnetic nanovectors increase apoptosis in glioblastoma cells through synergic intracellular hyperthermia and chemotherapy. Nanoscale 2019, 11, 72-88. [CrossRef]

31. Huszthy, P.C.; Daphu, I.; Niclou, S.P.; Stieber, D.; Nigro, J.M.; Sakariassen, P.Ø.; Miletic, H.; Thorsen, F.; Bjerkvig, R. In vivo models of primary brain tumors: Pitfalls and perspectives. Neuro-Oncol. 2012, 14, 979-993. [CrossRef] [PubMed]

32. Del Sol-Fernández, S.; Portilla-Tundidor, Y.; Gutiérrez, L.; Odio, O.F.; Reguera, E.; Barber, D.F.; Morales, M.P. Flower-like Mn-Doped Magnetic Nanoparticles Functionalized with $\alpha \mathrm{v} \beta 3$-Integrin-Ligand to Efficiently Induce Intracellular Heat after Alternating Magnetic Field Exposition, Triggering Glioma Cell Death. ACS Appl. Mater. Interfaces 2019, 11, 26648-26663. [CrossRef] [PubMed]

33. Herynek, V.; Turnovcová, K.; Veverka, P.; Dědourková, T.; Žvátora, P.; Jendelová, P.; Gálisová, A.; Kosinová, L.; Jiráková, K.; Syková, E. Using ferromagnetic nanoparticles with low curie temperature for magnetic resonance imaging-guided thermoablation. Int. J. Nanomed. 2016, 11, 3801-3811. [CrossRef] [PubMed]

34. Wu, V.M.; Huynh, E.; Tang, S.; Uskoković, V. Brain and bone cancer targeting by a ferrofluid composed of superparamagnetic iron-oxide/silica/carbon nanoparticles (earthicles). Acta Biomater. 2019, 88, $422-447$. [CrossRef]

35. Pernal, S.; Wu, V.M.; Uskoković, V. Hydroxyapatite as a Vehicle for the Selective Effect of Superparamagnetic Iron Oxide Nanoparticles against Human Glioblastoma Cells. ACS Appl. Mater. Interfaces 2017, 9, 39283-39302. [CrossRef] [PubMed]

36. Feuser, P.E.; Bubniak, L.D.S.; Silva, M.C.D.S.; Viegas, A.D.C.; Castilho Fernandes, A.; Ricci-Junior, E.; Nele, M.; Tedesco, A.C.; Sayer, C.; De Araújo, P.H.H. Encapsulation of magnetic nanoparticles in poly(methyl methacrylate) by miniemulsion and evaluation of hyperthermia in U87MG cells. Eur. Polym. J. 2015, 68, 355-365. [CrossRef]

37. Spirou, S.V.; Costa Lima, S.A.; Bouziotis, P.; Vranjes-Djuric, S.; Efthimiadou, E.; Laurenzana, A.; Barbosa, A.I.; Garcia-Alonso, I.; Jones, C.; Jankovic, D.; et al. Recommendations for In Vitro and In Vivo Testing of Magnetic Nanoparticle Hyperthermia Combined with Radiation Therapy. Nanomaterials 2018, 8, 306. [CrossRef]

38. Merle, P.; Camus, P.; Abergel, A.; Pageaux, G.P.; Masliah, C.; Bronowicki, J.P.; Zarski, J.P.; Pelletier, G.; Bouattour, M.; Farloux, L.; et al. Safety and efficacy of intra-arterial hepatic chemotherapy with doxorubicin-loaded nanoparticles in hepatocellular carcinoma. ESMO Open 2017, 2, e000238. [CrossRef]

39. Chen, Z.Y.; Wang, Y.X.; Lin, Y.; Zhang, J.S.; Yang, F.; Zhou, Q.L.; Liao, Y.Y. Advance of molecular imaging technology and targeted imaging agent in imaging and therapy. BioMed Res. Int. 2014, 2014, 819324. [CrossRef]

40. Pi, Z.; Huang, Y.; Shen, Y.; Zeng, X.; Hu, Y.; Chen, T.; Li, C.; Yu, H.; Chen, S.; Chen, X. Sonodynamic Therapy on Intracranial Glioblastoma Xenografts Using Sinoporphyrin Sodium Delivered by Ultrasound with Microbubbles. Ann. Biomed. Eng. 2019, 47, 549-562. [CrossRef]

41. Carvalho, L.A.; Teng, J.; Fleming, R.L.; Tabet, E.I.; Zinter, M.; de Melo Reis, R.A.; Tannous, B.A. Olfactory Ensheathing Cells: A Trojan Horse for Glioma Gene Therapy. J. Natl. Cancer Inst. 2019, 111, 283-291. [CrossRef] [PubMed]

42. Guerra-Rebollo, M.; Nogueira de Moraes, C.; Alcoholado, C.; Soler-Botija, C.; Sanchez-Cid, L.; Vila, O.F.; Meca-Cortes, O.; Ramos-Romero, S.; Rubio, N.; Becerra, J.; et al. Glioblastoma Bystander Cell Therapy: Improvements in Treatment and Insights into the Therapy Mechanisms. Mol. Ther. Oncolytics 2018, 11, 39-51. [CrossRef] [PubMed]

43. Meca-Cortes, O.; Guerra-Rebollo, M.; Garrido, C.; Borros, S.; Rubio, N.; Blanco, J. CRISPR/Cas9-Mediated Knockin Application in Cell Therapy: A Non-viral Procedure for Bystander Treatment of Glioma in Mice. Mol. Ther. Nucleic Acids 2017, 8, 395-403. [CrossRef] [PubMed]

44. Genevois, C.; Loiseau, H.; Couillaud, F. In Vivo Follow-up of Brain Tumor Growth via Bioluminescence Imaging and Fluorescence Tomography. Int. J. Mol. Sci. 2016, 17, 1815. [CrossRef] [PubMed]

45. Sanchez-Cabezas, S.; Montes-Robles, R.; Gallo, J.; Sancenon, F.; Martinez-Manez, R. Combining magnetic hyperthermia and dual T1/T2 MR imaging using highly versatile iron oxide nanoparticles. Dalton Trans. 2019, 48, 3883-3892. [CrossRef] [PubMed] 
46. Yao, J.; Hsu, C.H.; Li, Z.; Kim, T.S.; Hwang, L.P.; Lin, Y.C.; Lin, Y.Y. Magnetic resonance nano-theranostics for glioblastoma multiforme. Curr. Pharm. Des. 2015, 21, 5256-5266. [CrossRef]

47. Milanovic, D.; Braun, F.; Weber, W.; Grosu, A.L.; Behe, M.; Niedermann, G. The influence of the combined treatment with Vadimezan (ASA404) and taxol on the growth of U251 glioblastoma xenografts. BMC Cancer 2012, 12, 242. [CrossRef]

48. Liu, W.; Truillet, C.; Flavell, R.R.; Brewer, T.F.; Evans, M.J.; Wilson, D.M.; Chang, C.J. A reactivity-based [(18)F]FDG probe for in vivo formaldehyde imaging using positron emission tomography. Chem. Sci. 2016, 7, 5503-5507. [CrossRef]

49. Sha, W.; Ye, H.; Iwamoto, K.S.; Wong, K.P.; Wilks, M.Q.; Stout, D.; McBride, W.; Huang, S.C. Factors affecting tumor (18) F-FDG uptake in longitudinal mouse PET studies. EJNMMI Res. 2013, 3, 51. [CrossRef]

50. Van Dort, M.E.; Rehemtulla, A.; Ross, B.D. PET and SPECT Imaging of Tumor Biology: New Approaches towards Oncology Drug Discovery and Development. Curr. Comput. Aided Drug Des. 2008, 4, 46-53. [CrossRef]

51. Yu, E.Y.; Bishop, M.; Zheng, B.; Ferguson, R.M.; Khandhar, A.P.; Kemp, S.J.; Krishnan, K.M.; Goodwill, P.W.; Conolly, S.M. Magnetic Particle Imaging: A Novel in Vivo Imaging Platform for Cancer Detection. Nano Lett. 2017, 17, 1648-1654. [CrossRef] [PubMed]

52. Tay, Z.W.; Chandrasekharan, P.; Chiu-Lam, A.; Hensley, D.W.; Dhavalikar, R.; Zhou, X.Y.; Yu, E.Y.; Goodwill, P.W.; Zheng, B.; Rinaldi, C.; et al. Magnetic Particle Imaging-Guided Heating in Vivo Using Gradient Fields for Arbitrary Localization of Magnetic Hyperthermia Therapy. ACS Nano 2018, 12, 3699-3713. [CrossRef] [PubMed]

53. Souza, T.K.F.; Nucci, M.P.; Mamani, J.B.; da Silva, H.R.; Fantacini, D.M.C.; de Souza, L.E.B.; Picanco-Castro, V.; Covas, D.T.; Vidoto, E.L.; Tannus, A.; et al. Image and motor behavior for monitoring tumor growth in C6 glioma model. PLoS ONE 2018, 13, e0201453. [CrossRef] [PubMed]

54. Atif, F.; Yousuf, S.; Espinosa-Garcia, C.; Sergeeva, E.; Stein, D.G. Progesterone Treatment Attenuates Glycolytic Metabolism and Induces Senescence in Glioblastoma. Sci. Rep. 2019, 9, 988. [CrossRef] [PubMed]

55. Petty, R.D.; Sutherland, L.A.; Hunter, E.M.; Cree, I.A. Comparison of MTT and ATP-based assays for the measurement of viable cell number. J. Biolumin. Chemilumin. 1995, 10, 29-34. [CrossRef] [PubMed]

56. Magalhaes, C.M.; Esteves da Silva, J.C.; Pinto da Silva, L. Chemiluminescence and Bioluminescence as an Excitation Source in the Photodynamic Therapy of Cancer: A Critical Review. Chemphyschem Eur. J. Chem. Phys. Phys. Chem. 2016, 17, 2286-2294. [CrossRef]

57. Mezzanotte, L.; van't Root, M.; Karatas, H.; Goun, E.A.; Lowik, C. In Vivo Molecular Bioluminescence Imaging: New Tools and Applications. Trends Biotechnol. 2017, 35, 640-652. [CrossRef]

58. Viel, T.; Talasila, K.M.; Monfared, P.; Wang, J.; Jikeli, J.F.; Waerzeggers, Y.; Neumaier, B.; Backes, H.; Brekka, N.; Thorsen, F.; et al. Analysis of the growth dynamics of angiogenesis-dependent and -independent experimental glioblastomas by multimodal small-animal PET and MRI. J. Nucl. Med. Off. Publ. Soc. Nucl. Med. 2012, 53, 1135-1145. [CrossRef] [PubMed]

59. Donche, S.; Verhoeven, J.; Descamps, B.; Bolcaen, J.; Deblaere, K.; Boterberg, T.; Van den Broecke, C.; Vanhove, C.; Goethals, I. The Path Toward PET-Guided Radiation Therapy for Glioblastoma in Laboratory Animals: A Mini Review. Front. Med. 2019, 6, 5. [CrossRef]

60. Moreau, A.; Febvey, O.; Mognetti, T.; Frappaz, D.; Kryza, D. Contribution of Different Positron Emission Tomography Tracers in Glioma Management: Focus on Glioblastoma. Front. Oncol. 2019, 9, 1134. [CrossRef]

61. Bolcaen, J.; Acou, M.; Descamps, B.; Kersemans, K.; Deblaere, K.; Vanhove, C.; Goethals, I. PET for Therapy Response Assessment in Glioblastoma. In Glioblastoma; Codon Publications Copyright, De Vleeschouwer, S., Eds.; The Authors: Brisbane, Australia, 2017. [CrossRef]

62. Verger, A.; Langen, K.J. PET Imaging in Glioblastoma: Use in Clinical Practice. In Glioblastoma; Codon Publications Copyright, De Vleeschouwer, S., Eds.; The Authors: Brisbane, Australia, 2017. [CrossRef]

63. Aronen, H.J.; Pardo, F.S.; Kennedy, D.N.; Belliveau, J.W.; Packard, S.D.; Hsu, D.W.; Hochberg, F.H.; Fischman, A.J.; Rosen, B.R. High microvascular blood volume is associated with high glucose uptake and tumor angiogenesis in human gliomas. Clin. Cancer Res. Off. J. Am. Assoc. Cancer Res. 2000, 6, 2189-2200.

64. Mirus, M.; Tokalov, S.V.; Abramyuk, A.; Heinold, J.; Prochnow, V.; Zophel, K.; Kotzerke, J.; Abolmaali, N. Noninvasive assessment and quantification of tumor vascularization using [18F]FDG-PET/CT and CE-CT in a tumor model with modifiable angiogenesis-an animal experimental prospective cohort study. EJNMMI Res. 2019, 9, 55. [CrossRef] [PubMed] 
65. Toledo, M.; Busquets, S.; Sirisi, S.; Serpe, R.; Orpi, M.; Coutinho, J.; Martinez, R.; Lopez-Soriano, F.J.; Argiles, J.M. Cancer cachexia: Physical activity and muscle force in tumour-bearing rats. Oncol. Rep. 2011, 25, 189-193. [PubMed]

66. Stigliano, R.V.; Shubitidze, F.; Petryk, J.D.; Shoshiashvili, L.; Petryk, A.A.; Hoopes, P.J. Mitigation of eddy current heating during magnetic nanoparticle hyperthermia therapy. Int. J. Hyperth. 2016, 32, 735-748. [CrossRef] [PubMed]

67. Yameen, B.; Choi, W.I.; Vilos, C.; Swami, A.; Shi, J.; Farokhzad, O.C. Insight into nanoparticle cellular uptake and intracellular targeting. J. Control. Release 2014, 190, 485-499. [CrossRef]

68. Belanova, A.A.; Gavalas, N.; Makarenko, Y.M.; Belousova, M.M.; Soldatov, A.V.; Zolotukhin, P.V. Physicochemical Properties of Magnetic Nanoparticles: Implications for Biomedical Applications In Vitro and In Vivo. Oncol. Res. Treat. 2018, 41, 139-143. [CrossRef]

69. Rego, G.N.A.; Mamani, J.B.; Souza, T.K.F.; Nucci, M.P.; Silva, H.R.D.; Gamarra, L.F. Therapeutic evaluation of magnetic hyperthermia using Fe3O4-aminosilane-coated iron oxide nanoparticles in glioblastoma animal model. Einstein (Sao Paulo) 2019, 17, eAO4786. [CrossRef]

70. Youhannayee, M.; Nakhaei-Rad, S.; Haghighi, F.; Klauke, K.; Janiak, C.; Ahmadian, M.R.; Rabenalt, R.; Albers, P.; Getzlaff, M. Physical characterization and uptake of iron oxide nanoparticles of different prostate cancer cells. J. Magn. Magn. Mater. 2019, 473, 205-214. [CrossRef]

71. Shubitidze, F.; Kekalo, K.; Stigliano, R.; Baker, I. Magnetic nanoparticles with high specific absorption rate of electromagnetic energy at low field strength for hyperthermia therapy. J. Appl. Phys. 2015, 117, 094302. [CrossRef]

72. Kekalo, K.; Baker, I.; Meyers, R.; Shyong, J. Magnetic Nanoparticles with High Specific Absorption Rate at Low Alternating Magnetic Field. Nano Life 2015, 5. [CrossRef]

73. Yuan, Y.; Tasciuc, D.-A.B. Comparison between experimental and predicted specific absorption rate of functionalized iron oxide nanoparticle suspensions. J. Magn. Magn. Mater. 2011, 323, 2463-2469. [CrossRef]

74. Xu, H.; Pan, Y. Experimental Evaluation on the Heating Efficiency of Magnetoferritin Nanoparticles in an Alternating Magnetic Field. Nanomaterials 2019, 9, 1457. [CrossRef] [PubMed]

75. Ludwig, R.; Stapf, M.; Dutz, S.; Muller, R.; Teichgraber, U.; Hilger, I. Structural properties of magnetic nanoparticles determine their heating behavior-An estimation of the in vivo heating potential. Nanoscale Res. Lett. 2014, 9, 602. [CrossRef] [PubMed]

76. Murayama, S.; Nishiyama, T.; Takagi, K.; Ishizuka, F.; Santa, T.; Kato, M. Delivery, stabilization, and spatiotemporal activation of cargo molecules in cells with positively charged nanoparticles. Chem. Commun. 2012, 48, 11461-11463. [CrossRef]

77. Li, L.; Jiang, W.; Luo, K.; Song, H.; Lan, F.; Wu, Y.; Gu, Z. Superparamagnetic iron oxide nanoparticles as MRI contrast agents for non-invasive stem cell labeling and tracking. Theranostics 2013, 3, 595-615. [CrossRef]

78. Hu, L.; Mao, Z.; Gao, C. Colloidal particles for cellular uptake and delivery. J. Mater. Chem. 2009, 19, 3108-3115. [CrossRef]

79. Zhang, Y.; Yang, M.; Portney, N.G.; Cui, D.; Budak, G.; Ozbay, E.; Ozkan, M.; Ozkan, C.S. Zeta potential: A surface electrical characteristic to probe the interaction of nanoparticles with normal and cancer human breast epithelial cells. Biomed. Microdevices 2008, 10, 321-328. [CrossRef]

80. Bowman, C.L.; Lohr, J.W. Mechanotransducing ion channels in C6 glioma cells. Glia 1996, 18, 161-176. [CrossRef]

81. Rivet, C.J.; Yuan, Y.; Gilbert, R.J.; Borca-Tasciuc, D.A. Effect of magnetic nanoparticle heating on cortical neuron viability. Int. J. Hyperth. 2014, 30, 79-85. [CrossRef]

82. Zhong, L.; Chen, L.; Lv, S.; Li, Q.; Chen, G.; Luo, W.; Zhou, P.; Li, G. Efficacy of moderately hypofractionated simultaneous integrated boost intensity-modulated radiotherapy combined with temozolomide for the postoperative treatment of glioblastoma multiforme: A single-institution experience. Radiat. Oncol. 2019, 14, 104. [CrossRef]

83. Sulman, E.P.; Ismaila, N.; Armstrong, T.S.; Tsien, C.; Batchelor, T.T.; Cloughesy, T.; Galanis, E.; Gilbert, M.; Gondi, V.; Lovely, M.; et al. Radiation Therapy for Glioblastoma: American Society of Clinical Oncology Clinical Practice Guideline Endorsement of the American Society for Radiation Oncology Guideline. J. Clin. Oncol. Off. J. Am. Soc. Clin. Oncol. 2017, 35, 361-369. [CrossRef]

84. Grauer, O.; Jaber, M.; Hess, K.; Weckesser, M.; Schwindt, W.; Maring, S.; Wölfer, J.; Stummer, W. Combined intracavitary thermotherapy with iron oxide nanoparticles and radiotherapy as local treatment modality in recurrent glioblastoma patients. J. Neuro Oncol. 2019, 141, 83-94. [CrossRef] 
85. Jordan, A.; Scholz, R.; Maier-Hauff, K.; van Landeghem, F.K.H.; Waldoefner, N.; Teichgraeber, U.; Pinkernelle, J.; Bruhn, H.; Neumann, F.; Thiesen, B.; et al. The effect of thermotherapy using magnetic nanoparticles on rat malignant glioma. J. Neuro Oncol. 2006, 78, 7-14. [CrossRef]

86. James, M.L.; Gambhir, S.S. A molecular imaging primer: Modalities, imaging agents, and applications. Physiol. Rev. 2012, 92, 897-965. [CrossRef] [PubMed]

87. Frosina, G. Positron emission tomography of high-grade gliomas. J. Neurooncol. 2016, 127, 415-425. [CrossRef] [PubMed]

88. Fischer, B.M.; Olsen, M.W.; Ley, C.D.; Klausen, T.L.; Mortensen, J.; Hojgaard, L.; Kristjansen, P.E. How few cancer cells can be detected by positron emission tomography? A frequent question addressed by an in vitro study. Eur. J. Nucl. Med. Mol. Imaging 2006, 33, 697-702. [CrossRef]

89. Warnock, G.; Turtoi, A.; Blomme, A.; Bretin, F.; Bahri, M.A.; Lemaire, C.; Libert, L.C.; Seret, A.E.; Luxen, A.; Castronovo, V.; et al. In vivo PET/CT in a human glioblastoma chicken chorioallantoic membrane model: A new tool for oncology and radiotracer development. J. Nucl. Med. Off. Publ. Soc. Nucl. Med. 2013, 54, 1782-1788. [CrossRef]

90. Alf, M.F.; Wyss, M.T.; Buck, A.; Weber, B.; Schibli, R.; Kramer, S.D. Quantification of brain glucose metabolism by 18 F-FDG PET with real-time arterial and image-derived input function in mice. J. Nucl. Med. Off. Publ. Soc. Nucl. Med. 2013, 54, 132-138. [CrossRef]

91. Colavolpe, C.; Chinot, O.; Metellus, P.; Mancini, J.; Barrie, M.; Bequet-Boucard, C.; Tabouret, E.; Mundler, O.; Figarella-Branger, D.; Guedj, E. FDG-PET predicts survival in recurrent high-grade gliomas treated with bevacizumab and irinotecan. Neuro Oncol. 2012, 14, 649-657. [CrossRef]

92. Paxinos, G.; Watson, C. The Rat Brain in Stereotaxic Coordinates; Acad. Press: Cambridge, MA, USA, 1986.

(C) 2020 by the authors. Licensee MDPI, Basel, Switzerland. This article is an open access article distributed under the terms and conditions of the Creative Commons Attribution (CC BY) license (http://creativecommons.org/licenses/by/4.0/). 